\title{
Modeling the Macrophage-Mediated Inflammation Involved in the Bone Fracture Healing Process
}

\author{
Imelda Trejo *(1), Hristo Kojouharov and Benito Chen-Charpentier (1) \\ Department of Mathematics, The University of Texas at Arlington, P.O. Box 19408, \\ Arlington, TX 76019-0408, USA; hristo@uta.edu (H.K.); bmchen@uta.edu (B.C.-C.) \\ * Correspondence: imelda.trejo@mavs.uta.edu; Tel.: +1-817-272-3261
}

Received: 14 November 2018; Accepted: 15 January 2019; Published: 17 January 2019

check for updates

\begin{abstract}
A new mathematical model is presented to study the effects of macrophages on the bone fracture healing process. The model consists of a system of nonlinear ordinary differential equations that represents the interactions among classically and alternatively activated macrophages, mesenchymal stem cells, osteoblasts, and pro- and anti-inflammatory cytokines. A qualitative analysis of the model is performed to determine the equilibria and their corresponding stability properties. Numerical simulations are also presented to support the theoretical results, and to monitor the evolution of a broken bone for different types of fractures under various medical interventions. The model can be used to guide clinical experiments and to explore possible medical treatments that accelerate the bone fracture healing process, either by surgical interventions or drug administrations.
\end{abstract}

Keywords: bone repair; macrophages; immune system; cytokines; stem cells

\section{Introduction}

Bone fractures are becoming a serious worldwide problem, due to their high frequency and surgical complications. Globally, more than 8.9 million fractures occur every year, where $10-15 \%$ of them result in nonunion [1-5]. Prolonged healing, disabilities, and high morbidity rates are associated with severe traumas and immune-compromised-fractured people [2,3,6-8]. In addition, medical care costs for bone fractures are expected to be over US $\$ 25$ billions by 2025 ; due, in part, to the expensive treatments and prolonged hospitalization and rehabilitation [3,9]. It is essential to have a better understanding of the bone fracture healing process, in order to prevent unsuccessful healing and to develop optimal fracture union treatments.

Recently, experimental and mathematical models have demonstrated that macrophages strongly regulate bone fracture healing [10,11]. Depletion of macrophages results in delayed bone formation [12]. Furthermore, during inflammation, classically activated macrophages attract mesenchymal lineage cells (MSCs) to the injury site and activate the healing process [3,10,12]. In contrast, during the repair phase, alternatively activated macrophages promote MSC proliferation and differentiation, and accelerate bone healing $[10,13]$. However, the exact mechanisms by which macrophages contribute to bone healing remain unclear $[3,10,14]$. Also, the interaction between macrophages and tissue cells, as well as the importance of classically and alternatively activated macrophages during the bone healing process, are still not clearly understood $[10,12,13]$.

In [11], a mathematical model, based on the interactions among macrophages, MSCs, and osteoblasts, was developed to study the regulatory effects of two generic pro- and anti-inflammatory cytokines during the early stages of bone fracture healing. To our knowledge, it was the first attempt to incorporate the macrophage interactions in the modeling of the bone fracture healing process. The mathematical model revealed that high concentrations of pro-inflammatory cytokines negatively affect the healing time of a fracture, while the administration of anti-inflammatory cytokines can accelerate the healing time in a 
dose-dependent manner. Therefore, it is important to carefully consider and incorporate in the modeling approach all sources of pro- and anti-inflammatory cytokines, such as macrophages [2,3,15], in order to correctly represent the complex progression of the bone fracture healing process.

In this paper, the mathematical model developed in [11] is extended, to separately incorporate the two different phenotypes of macrophages: Classically and alternatively activated macrophages, as they have distinct functions during the healing process $[10,15,16]$. Classically activated macrophages release high levels of pro-inflammatory cytokines, including TNF- $\alpha$ and IL- $1 \beta$, which exhibit inhibitory and destructive properties in high concentrations $[16,17]$. In contrast, alternatively activated macrophages are characterized by the secretion of anti-inflammatory cytokines, such as IL-10, which increase their phagocytic activities, mitigate the inflammatory responses, promote growth, and accelerate fracture healing $[2,3,15,16]$. This extension leads to a more realistic model, by incorporating the different phagocytic rates and the separate production of the pro- and anti-inflammatory cytokines by the two types of macrophages $[15,18]$. The model can be used to investigate the macrophage functions during inflammation and their effects during the bone fracture healing process. The model can also be used to investigate potential therapeutic treatments, based on the use of anti-inflammatory cytokines, stem cells, and macrophages, suggesting possible ways to guide clinical experiments and bone tissue engineering strategies $[15,16]$.

The organization of the paper is as follows: Section 2 discusses the cellular and molecular interactions that occur during the bone fracture healing process. The macrophage-mediated inflammation involved in the bone fracture healing process is also described in detail. The simplifying assumptions are presented in Section 3. In Section 4, a system of nonlinear ordinary differential equations is introduced, to mathematically describe the fundamental aspects of the bone fracture healing process during the resolution of inflammation and bone repair. The stability analysis of the system is presented in Section 5 . Section 6 demonstrates the functionality of the model, by numerically simulating the progression of the bone fracture healing process under normal and pathological conditions. The discussion and future work are presented in Section 7.

\section{Biological Background}

Bone fracture healing is a complex biological process, which involves the participation of different cell types (including the immune system and mesenchymal lineage cells [16]) and is strongly regulated by released molecular factors [10,16,19-21]. Particularly, at the beginning of the healing process, cytokines activate and direct both immune and tissue cellular functions.

Cytokines are functionally classified into pro-inflammatory and anti-inflammatory families. Pro-inflammatory cytokines, such as the tumor necrotic factor- $\alpha$ (TNF- $\alpha$ ), activate the immune system defense to kill bacteria and fight infections. Anti-inflammatory cytokines block the pro-inflammatory synthesis and activate the mesenchymal lineage cellular functions [2]. Interleukin-10 (IL-10) is one of the most potent anti-inflammatory molecules that inhibits pro-inflammatory production [2,22], and is mainly delivered by macrophages and MSCs [2]. A correct balance between pro- and anti-inflammatory cytokines during fracture healing is necessary for successful fracture repair. High levels of TNF- $\alpha$ induce chronic inflammation and gradual destruction of cartilage and bone tissue [21], while the absence of TNF- $\alpha$ results in nonunion or delayed nonunions $[17,23]$.

Bone fracture healing can be described in three characteristic phases: Inflammatory, repair, and remodelling (see Figure 1) [24]. During inflammation, necroses of cells result in the delivery of pro-inflammatory cytokines which attract inflammatory immune cells, such as neutrophils and monocytes [1,23], to the injury site. In response to their phagocytic activities, these cells magnify the pro-inflammatory production, leading to an acute inflammation $[17,23,25]$. Subsequently, monocytes differentiate into macrophages to down-regulate the inflammation and resolve it. Once this differentiation begins, the influx of the inflammatory cells ceases, and they die out [26].

During the resolution of inflammation, macrophages increase their population by migration and activate to their classical and alternative phenotypes, accordingly to the cytokine stimuli $[16,27]$. 
The two phenotypes can also shift between each other during this process [28,29]. Macrophages have the capability to release both pro- and anti-inflammatory cytokines through their different phenotypes [28]. Classically activated macrophages release high concentration of pro-inflammatory cytokines, including TNF- $\alpha$, and low levels of anti-inflammatory cytokines [28], in response to their engulfing functions. Alternatively activated macrophages secrete high levels of Il-10 and low levels of TNF- $\alpha$, as they continue with the clearance of debris and the modulation of inflammation [28].

During the repair phase, migrating MSCs contribute with the delivery of IL-10, and proliferate or differentiate into fibroblasts, chondrocytes, and osteoblasts [2,30]. Fibroblasts and chondrocytes proliferate and release the fibrinous/cartilagenous extracellular matrix, which fills up the fracture gap $[10,30,31]$ while osteoblasts proliferate and deposit the new bone, also called woven bone [30]. Bone deposit results from mineralized collagen and other proteins being delivered by the osteoblasts [10]. After bone mineralization, osteoblasts remain on the bone surface or differentiate into osteocytes, which become part of the bone extracellular matrix [32,33].

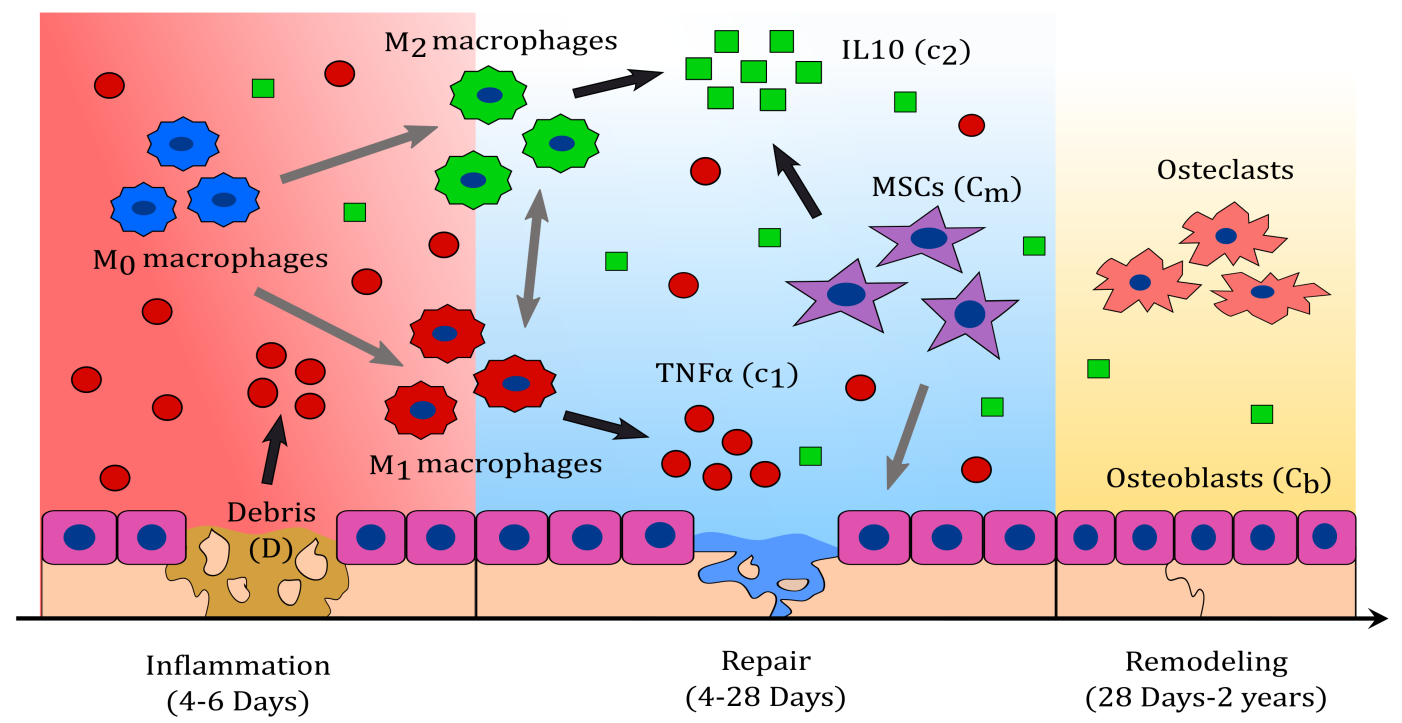

Figure 1. Inflammatory, repair, and remodeling phases of the bone fracture healing process. During the inflammatory phase, debris $(D)$ activates the healing process by attracting macrophages $M_{0}$ to the injury site, which subsequently activate into their $M_{1}$ or $M_{2}$ phenotypes. Activated macrophages remove debris and secrete pro- and anti-inflammatory cytokines, such as tumor necrotic factor- $\alpha$ $(\mathrm{TNF}-\alpha)\left(c_{1}\right)$ and interleukin-10 (IL-10) $\left(c_{2}\right)$, which regulate the inflammation and the cellular functions. During the repair phase, migrating mesenchymal stem cells (MSCs) up-regulate IL-10 production, proliferate, and differentiate into osteoblasts $\left(C_{b}\right)$. Mesenchymal and osteoblast cells synthesize the fibro/cartilage and woven bone, which closes the fracture gap. During the bone remodeling phase, osteoblasts and osteoclasts constantly remove and deposit new bone until the fracture is fully repaired.

During the last phase of the bone fracture healing process, the fibrocartilage and the woven bone are constantly removed and replaced by a functional bone [34]. This process is referred to as bone remodeling, and consists of systematic tissue degradation and production by osteoclasts and osteoblasts, respectively. Bone remodeling is a slow process, that can take months to years until the bone recovers to its pre-injury state [14]. In a moderate fracture, acute inflammation is observed $24 \mathrm{~h}$ after the injury; it also corresponds to the peak of TNF- $\alpha$, which returns to baseline levels within $72 \mathrm{~h}[14,23]$. Fibrinous/cartilaginous tissue production is observed in the first 3 days, peaks in about 10 to 12 days, and its removal starts as early as 21 days [30]. The inflammation is considered resolved when the debris is eliminated, activated macrophages emigrate to the lymphatic nodes to die, and inactivated macrophages return to their normal density [26]. These events are observed after two weeks from the beginning of the healing process [34,35]. At approximately 28 to 35 days, osteoclasts populate the injury site and a substantial removal of the fibrocartilage is observed [34]. The fracture healing 
outcome is considered a delayed union if the fibrous/cartilaginous tissue is not removed completely in about 3 to 4 months after the injury, while it is considered a nonunion if no functional bone is obtained in 6 months after the trauma [36].

\section{Modeling Assumptions}

The most important effects of macrophages on bone fracture healing are observed during the inflammatory and repair phases of the healing process [11,16]. During the inflammatory phase, macrophages modulate and resolve the inflammation while, during the repair phase, macrophages provide an optimal environment for the cellular proliferation, differentiation, and tissue production. The primary variables during the inflammatory and repair phases of the bone fracture healing process are debris $(D)$, unactivated macrophages $\left(M_{0}\right)$, classical macrophages $\left(M_{1}\right)$, alternative macrophages $\left(M_{2}\right)$, MSCs $\left(C_{m}\right)$, osteoblasts $\left(C_{b}\right)$, pro-inflammatory cytokines $\left(c_{1}\right)$, anti-inflammatory cytokines $\left(c_{2}\right)$, fibrocartilage $\left(m_{c}\right)$, and woven bone $\left(m_{b}\right)$.

The biological system interactions are depicted in Figure 2. The cells and cellular dynamics are represented by the circular shapes and solid arrows. The molecular concentrations and their production/decay are represented by the octagonal shapes and dashed arrows. The pro- and anti-inflammatory cytokine activation/inhibition effects on the cellular functions are represented by the dotted arrows. Removal of debris and the negative effect among the variables are represented by the dot-ending dotted arrows.

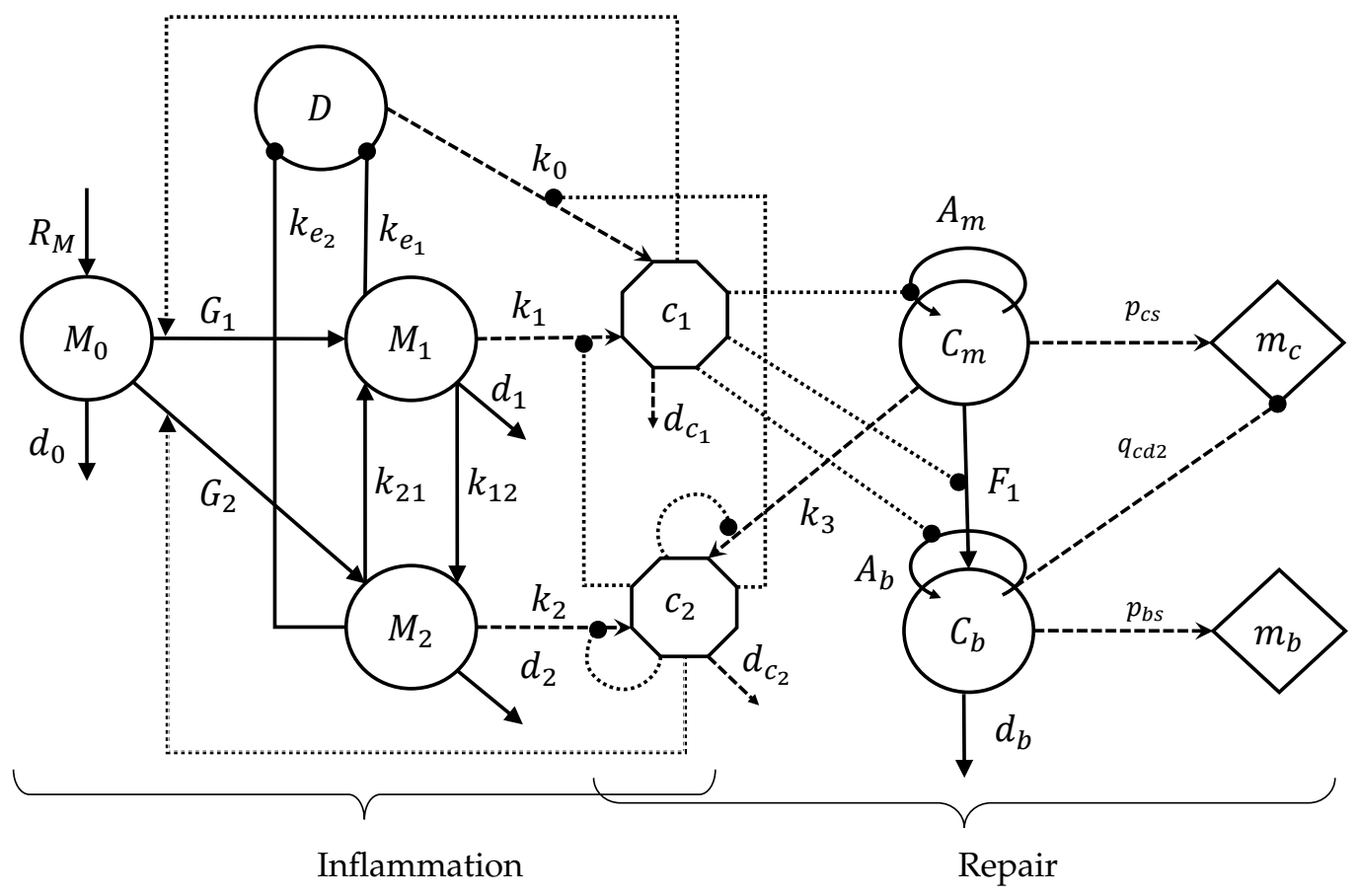

Figure 2. Flow diagram of the cellular and molecular dynamics during the inflammatory and repair phases of the bone fracture healing process.

It is assumed that the cellular functions are regulated by $c_{1}$ (such as TNF- $\alpha$ ) and $c_{2}$ (such as IL-10). It is also assumed that $c_{1}$ is delivered through cell necrosis and by the classically activated macrophages, while $c_{2}$ is delivered by the alternatively activated macrophages and MSCs. It is further assumed that the repair process is governed by the production of $m_{c}$ and $m_{b}$ [30,37], whose final levels are used to classify the outcome of the bone healing process. Additionally, it is assumed that the debris $D$ are proportional to the number of necrotic cells [11]. It is also assumed that unactivated macrophages $M_{0}$ do not release cytokines and do not engulf debris. Additionally, the population of $M_{0}$ increases proportionally in size to the density of debris, up to a maximal value of $M_{\max }$ [27]. The only 
source of activated macrophages, $M_{1}$ and $M_{2}$, is $M_{0}$. Even though both phenotypes of activated macrophages have the ability to release both pro- and anti-inflammatory cytokines, it is assumed that only $M_{1}$ deliver $c_{1}$ and $M_{2}$ deliver $c_{2}$, as those are the major cytokines for each phenotype [38]. $M_{0}$ activate to $M_{1}$ under the $c_{1}$ stimulus, while they activate to $M_{2}$ under the $c_{2}$ stimulus. $M_{1}$ and $M_{2}$ macrophages do not de-differentiate back to the $M_{0}$ macrophages [39], and are able to switch phenotypes at a constant rate [29]. The accumulation of macrophages at the injury site is modeled by its recruitment due to inflammation, which is assumed to be proportional to the debris density.

Furthermore, it is assumed that the differentiation rates of MSCs into osteoblasts and osteoblasts into osteocytes are constant. MSCs synthesize the fibrocartilage, while osteoblasts synthesize the woven bone. It is also assumed that only the fibrocartilage is constantly removed by the osteoclasts, with the density of the osteoclasts being assumed proportional to the density of the osteoblasts [30]. In addition, it is assumed that the populations of the two tissue cells, $C_{m}$ and $C_{b}$, experience logistic growth, where the growth rates decrease linearly as the population sizes approach a maximum value, $K_{l m}$ and $K_{l b}$, respectively, imposed by the limited resources of the environment [30,40]. It is also assumed that there is no recruitment of MSCs and osteoblasts.

\section{Model Formulation}

The inflammatory and repair phases of the bone fracture healing process are modeled with a mass-action system of nonlinear ordinary differential equations. All variables represent homogeneous quantities in a given volume. Following the outlined biological assumptions and the flow diagram given in Figure 2 yields the resulting system of equations:

$$
\begin{aligned}
\frac{d D}{d t} & =-R_{D}\left(k_{e_{1}} M_{1}+k_{e_{2}} M_{2}\right) \\
\frac{d M_{0}}{d t} & =R_{M}-G_{1} M_{0}-G_{2} M_{0}-d_{0} M_{0} \\
\frac{d M_{1}}{d t} & =G_{1} M_{0}+k_{21} M_{2}-k_{12} M_{1}-d_{1} M_{1} \\
\frac{d M_{2}}{d t} & =G_{2} M_{0}+k_{12} M_{1}-k_{21} M_{2}-d_{2} M_{2} \\
\frac{d c_{1}}{d t} & =H_{1}\left(k_{0} D+k_{1} M_{1}\right)-d_{c_{1}} c_{1} \\
\frac{d c_{2}}{d t} & =H_{2}\left(k_{2} M_{2}+k_{3} C_{m}\right)-d_{c_{2}} c_{2} \\
\frac{d C_{m}}{d t} & =A_{m} C_{m}\left(1-\frac{C_{m}}{K_{l m}}\right)-F_{1} C_{m} \\
\frac{d C_{b}}{d t} & =A_{b} C_{b}\left(1-\frac{C_{b}}{K_{l b}}\right)+F_{1} C_{m}-d_{b} C_{b} \\
\frac{d m_{c}}{d t} & =\left(p_{c s}-q_{c d 1} m_{c}\right) C_{m}-q_{c d 2} m_{c} C_{b} \\
\frac{d m_{b}}{d t} & =\left(p_{b s}-q_{b d} m_{b}\right) C_{b}
\end{aligned}
$$

Equation (1) describes the rate of change with respect to time of the debris density, which decreases proportionally to $M_{1}$ and $M_{2}$. The engulfing rate $R_{D}$ is modeled by a Hill Type II function to represent the saturation of the phagocyte rate of macrophages $[38,41]$ :

$$
R_{D}=\frac{D}{a_{e d}+D} .
$$

Equation (2) describes the rate of change with respect to time of the undifferentiated macrophages density. It increases because of migration and decreases by differentiating into $M_{1}$ and $M_{2}$ or by a 
constant emigration rate. It is assumed that $M_{0}$ migrate to the injury site proportionally to $D$, up to a maximal constant rate, $k_{\max },[22,28]$ :

$$
R_{M}=k_{\max }\left(1-\frac{M}{M_{\max }}\right) D,
$$

where $M=M_{0}+M_{1}+M_{2}$. The differentiation rates of $M_{0}$ into $M_{1}$ and $M_{2}$ are stimulated by the cytokines accordingly to Hill Type II equations, respectively [29]:

$$
G_{1}=k_{01} \times \frac{c_{1}}{a_{01}+c_{1}}, \quad G_{2}=k_{02} \times \frac{c_{2}}{a_{02}+c_{2}} .
$$

Equation (3) describes the rate of change with respect to time of $M_{1}$, which increases when $M_{0}$ activates to $M_{1}$, and $M_{2}$ shifts phenotype; and decreases by emigration, and when $M_{1}$ shift phenotype. Similarly, Equation (4) describes the rate of change with respect to time of $M_{2}$. Equations (5) and (6) describes the rate of change with respect to time of $c_{1}$ and $c_{2}$. Here, $k_{0}, k_{1}, k_{2}$, and $k_{3}$ are the constant rates of the cytokine productions and $d_{c_{1}}$ and $d_{c_{2}}$ are the cytokine constant decay rates. The inhibitory effects of the anti-inflammatory cytokines are modeled by the following functions [29]:

$$
H_{1}=\frac{a_{12}}{a_{12}+c_{2}}, \quad H_{2}=\frac{a_{22}}{a_{22}+c_{2}} .
$$

Equation (7) describes the rate of change with respect to time of $C_{m}$, which increases by cellular division up to a constant-maximal carrying capacity, $K_{l m}$, and decreases by differentiation [30]. The total MSC proliferation rate is modeled by [42]:

$$
A_{m}=k_{p m} \times \frac{a_{p m}^{2}+a_{p m_{1}} c_{1}}{a_{p m}^{2}+c_{1}^{2}},
$$

where in the absent of inflammation, $c_{1}=0$, MSC proliferate at a constant rate $k_{p m}$. However, when there is inflammation, $c_{1}>0$, and the proliferation rate of MSCs increases or decreases according to the concentration of $c_{1}$ (i.e., high concentration levels of $c_{1}$ inhibit $C_{m}$ proliferation, while low concentration levels of $c_{1}$ accelerate $C_{m}$ proliferation). The differentiation rate of $C_{m}$ is inhibited by $c_{1}$, which is modeled by the following function [11]:

$$
F_{1}=d_{m} \times \frac{a_{m b_{1}}}{a_{m b_{1}}+c_{1}} .
$$

Equation (8) describes the rate of change with respect to time of $C_{b}$. It increases when MSC differentiate into osteoblasts, or when osteoblasts proliferate [30]. It decreases at a constant rate $d_{b}$ when osteoblasts differentiate into osteocytes. The osteoblast proliferation rate is inhibited by $c_{1}$, which is modeled by the following function [11]:

$$
A_{b}=k_{p b} \times \frac{a_{p b}}{a_{p b}+c_{1}} .
$$

Equations (9) and (10) describe the rate of change with respect to time of the fibrocartilage and woven bone, where $p_{c s}$ and $p_{b s}$ are the tissue constant synthesis rates and $q_{c d 1}, q_{c d 2}$, and $q_{b d}$ are the tissue degradation rates, respectively [30]. 


\section{Analysis of the Model}

The analysis of Model (1)-(10) is done by finding the equilibria and their corresponding stability properties. An equilibrium is a state of the system where the variables do not change over time [40]. Once the equilibria are identified, it is important to determine the behavior of the model near the equilibria by analyzing their local stability properties. An equilibrium is locally stable if the system moves toward it when it is near the equilibrium, otherwise it is unstable [40]. Therefore, the equilibria provide the possible outcomes of the bone fracture healing process, and their corresponding stability properties define the conditions under which a particular healing result occurs.

System (1)-(10) has the following three biologically meaningful equilibria of the vector form $E=$ $\left(D, M_{0}, M_{1}, M_{2}, c_{1}, c_{2}, C_{m}, C_{b}, m_{c}, m_{b}\right): E_{0}=\left(0,0,0,0,0,0,0,0, m_{c_{0}}^{*}, m_{b_{0}}^{*}\right), E_{1}=\left(0,0,0,0,0,0,0, K_{l b}(1-\right.$ $\left.\left.d_{b} / k_{p b}\right), 0, p_{b s} / q_{b d}\right), E_{2}=\left(0,0,0,0,0, c_{2}^{*}, C_{m}^{*}, C_{b}^{*}, m_{c}^{*}, p_{b s} / q_{b d}\right)$, where $C_{m}^{*}=K_{l m}\left(1-d_{m} / k_{p m}\right), C_{b}^{*}=$ $K_{l b}\left(k_{p b}-d_{b}+\sqrt{\left(k_{p b}-d_{b}\right)^{2}+4 k_{p b} d_{m} C_{m}^{*} / K_{l b}}\right) / 2 k_{p b}, c_{2}^{*}=a_{22}\left(-1+\sqrt{1+4 k_{3} C_{m}^{*} / a_{22} d_{c_{2}}}\right) / 2$, and $m_{c}^{*}=p_{c s} C_{m}^{*} /\left(q_{c d 1} C_{m}^{*}+q_{c d 2} C_{b}^{*}\right)$. The existence conditions for the three equilibria are summarized in Table 1 and their stability conditions are summarized in Table 2, and are proved in Appendix A.

The existence conditions, listed in Table 1 , arise from the fact that all biologically meaningful variables are nonnegative. Therefore, the existence condition for $E_{0}$ requires the steady state tissue densities to be either zero or any positive number. For $E_{1}$, the existence condition arises from the requirement that the steady state density of $C_{b}$ must be greater than zero, which implies that the proliferation rate of osteoblasts must be greater than their differentiation rate (i.e., $k_{p b}>d_{b}$ ).

Similarly for $E_{2}$, the existence condition arises from the requirement that the steady state density for $C_{m}$ must be greater than zero, which implies that the proliferation rate of MSCs must be greater than their differentiation rate (i.e., $k_{p m}>d_{m}$ ).

Table 1. Existence conditions for the equilibrium points and their biological meaning.

\begin{tabular}{ccc}
\hline Equilibrium Points & Existence Conditions & Meaning \\
\hline$E_{0}=\left(0,0,0,0,0,0,0,0, m_{c_{0}}^{*}, m_{b_{0}}^{*}\right)$ & $m_{c_{0}}^{*} \geq 0, m_{b_{0}}^{*} \geq 0$ & nonunion \\
$E_{1}=\left(0,0,0,0,0,0,0, K_{l b}\left(1-d_{b} / k_{p b}\right), 0, p_{b s} / q_{b d}\right)$ & $k_{p b}>d_{b}$ & successful healing \\
$E_{2}=\left(0,0,0,0,0, c_{2}^{*}, C_{m}^{*}, C_{b}^{*}, m_{c}^{*}, p_{b s} / q_{b d}\right)$ & $k_{p m}>d_{m}$ & nonunion or delayed union \\
\hline
\end{tabular}

The stability conditions of each biologically feasible equilibrium are listed in Table 2, and is determined from the eigenvalues of its associated Jacobian matrix (see Appendix $A$ ), as follows:

$E_{0}$ is stable when $k_{p m} \leq d_{m}$ and $k_{p b} \leq d_{b}$ (see Theorem A1), which implies that the differentiation rates of the MSC and osteoblasts are greater than or equal to their proliferation rates, respectively. The steady-state $E_{0}$ represents a nonunion. In this case, the inflammation is resolved, since the first five entries of $E_{0}$ are zero; however, the repair process has failed since the osteoblasts and osteoclasts have died out before the beginning of the remodeling process. Hence, the tissue densities, $m_{c_{0}}^{*}$ and $m_{b_{0}}^{*}$ can be any two positive values smaller than their maximal densities, $p_{c s} / q_{c d 1}$ and $p_{b s} / q_{b d}$, respectively (see Theorem A1).

$E_{1}$ is stable when $k_{p m} \leq d_{m}$ and $k_{p b}>d_{b}$ (see Theorem A2). The steady-state $E_{1}$ represents a successful repair of the bone fracture, where the inflammation is resolved, the fibrocartilage is completely removed from the repair site, and the woven bone has achieved its maximal density. In this case, osteoblasts proliferate faster than they differentiate, while MSC have the opposite behavior.

$E_{2}$ is stable when $k_{p m}>d_{m}$ (see Theorem A3). The steady-state $E_{2}$ represents a nonunion or delayed union, where the inflammation is resolved, but the osteoclasts have failed to degrade the cartilage in a timely fashion. 
Table 2. Stability conditions for the equilibrium points.

\begin{tabular}{ccc}
\hline Equilibrium Points & Stability Conditions & Stability \\
\hline$E_{0}$ & $k_{p m} \leq d_{m}, k_{p b} \leq d_{b}$ & $E_{0}$ belongs to an attracting local set \\
$E_{0}, E_{1}$ & $k_{p m} \leq d_{m}, k_{p b}>d_{b}$ & $E_{0}$ unstable; $E_{1}$ locally stable \\
$E_{0}, E_{2}$ & $k_{p m}>d_{m}, k_{p b} \leq d_{b}$ & $E_{0}$ unstable; $E_{2}$ locally stable \\
$E_{0}, E_{1}, E_{2}$ & $k_{p m}>d_{m}, k_{p b}>d_{b}$ & $E_{0}$ and $E_{1}$ unstable; $E_{2}$ locally stable \\
\hline
\end{tabular}

\section{Numerical Results}

The proposed new model (1)-(10) is used to study the importance of macrophages during the inflammatory and repair phases of the bone fracture healing process, which occur within the first 21 days after trauma [11,13]. It is also used to investigate the evolution of a broken bone under normal and pathological conditions. Table 3 summarizes the baseline parameter values and units for the numerical simulations. These values are estimated in a qualitative manner from data in other studies $[11,27,30,38,39,43]$. Some of those, from [11], were also rescaled to account for the different mathematical expressions of the proliferation and differentiation rates of the tissue cells. All parameter values are based on murine experiments, with healthy mice having a moderate fracture (a broken bone with a gap size less than $3 \mathrm{~mm}$ ) [30,43]. However, the bone fracture healing process for humans involves the same cells, cytokines, and qualitative dynamics, differing only in the number of cells, concentrations, and the length of time it takes for a full recovery [24].

First, a set of numerical simulation results was presented to compare two mathematical models of the bone fracture healing process that incorporate macrophages: The model developed in [11], and the new model (1)-(10). Next, numerical simulations were performed to support the theoretical stability results (successful and nonunion equilibria) and to numerically monitor the healing progression of a moderate fracture in normal conditions. Another set of numerical simulations was performed to analyze the effects of different debris densities on bone fracture healing. Finally, a set of numerical simulation results was presented, to investigate the effects of different concentrations of anti-inflammatory cytokines and various cellular treatments on the fracture healing under numerous pathological conditions. All simulations were obtained by using the adaptive MATLAB solver ode23s, and were initiated with densities of debris, macrophages, and MSCs set to $D(0)=5 \times 10^{7}$, $M_{0}(0)=4000, C_{m}(0)=1000$, respectively, and the pro-inflammatory cytokine concentration set to $c_{1}(0)=1$.

\subsection{Comparison of Existing Models}

The model developed in [11] and the present mathematical model (1)-(10) were compared when $D(0)<a_{e d}=4.71 \times 10^{6}$ (i.e., the initial debris concentration was below the half-saturation of debris). In this case, the macrophage digestion rate increased approximately linearly with respect to the debris population, as assumed in model [11]. The same parameter values were used in both models (Table 3), with $k_{e_{1}}=11, k_{e_{2}}=48, k_{2}=3.72 \times 10^{-6}, k_{3}=8 \times 10^{-6}$, and $q_{b d}=5 \times 10^{-8}$.

Figure 3 shows the numerical evolutions of the tissues' production when $D(0)=2 \times 10^{6}$. In all simulations, we refer to fibrocartilage and woven bone as cartilage and bone, respectively. The production of cartilage $m_{c}$ and bone $m_{b}$ given by the present model is much more realistic than the production given by the model developed [11], since, according to the experimental data, the cartilage production peaks to its maximal density of around $1 \mathrm{~g} / \mathrm{mL}$ about $10-12$ days after trauma, and significant bone tissue production is observed after the second week [44]. 
Table 3. Parameter descriptions and units.

\begin{tabular}{|c|c|c|c|}
\hline Parameter & Description & Range of Values & Reference \\
\hline$k_{e_{1}}$ & Engulfing debris rate of $M_{1}$ & $3-48 /$ day & {$[38,41]$} \\
\hline$k_{e_{2}}$ & Engulfing debris rate of $M_{2}$ & 3-48/day & {$[38,41]$} \\
\hline$a_{e d}$ & Half-saturation of debris & $4.71 \times 10^{6}$ cells $/ \mathrm{mL}$ & [38] \\
\hline$k_{\max }$ & Maximal migration rate & $0.015-0.1 /$ day & {$[39,45]$} \\
\hline$M_{\max }$ & Maximal macrophages density & $6 \times 10^{5}-1 \times 10^{6}$ cells $/ \mathrm{mL}$ & {$[27,41]$} \\
\hline$k_{01}$ & Activation rate of $M_{1}$ & $0.55-0.611 /$ day & {$[29,39]$} \\
\hline$k_{02}$ & Activation rate of $M_{0}$ to $M_{2}$ & $0.0843-0.3 /$ day & [29] \\
\hline$k_{12}$ & Transition rate from $M_{1}$ to $M_{2}$ & $0.083-0.075 /$ day & {$[29,39]$} \\
\hline$k_{21}$ & Transition rate from $M_{2}$ to $M_{1}$ & $0.005-0.05 /$ day & [29] \\
\hline$d_{0}$ & Emigration rate of $M_{0}$ & $0.156-0.02 /$ day & {$[29,39]$} \\
\hline$d_{1}$ & Emigration rate of $M_{1}$ & $0.121-0.2 /$ day & {$[29,38,39]$} \\
\hline$d_{2}$ & Emigration rate of $M_{2}$ & $0.163-0.2 /$ day & {$[29,38,39]$} \\
\hline$k_{0}$ & Secretion rate of $c_{1}$ by debris & $5 \times 10^{-7}-8.5 \times 10^{-6} \mathrm{ng} /$ cells $/$ day & [38] \\
\hline$k_{1}$ & Secretion rate of $c_{1}$ by $M_{1}$ macrophages & $8.3 \times 10^{-6} \mathrm{ng} /$ cells $/$ day & [38] \\
\hline$k_{2}$ & Secretion rate of $c_{2}$ by $M_{2}$ macrophages & $3.72 \times 10^{-6} \mathrm{ng} /$ cells / day & [38] \\
\hline$k_{3}$ & Secretion rate of $c_{2}$ by MSCs & $7 \times 10^{-7}-8 \times 10^{-6} \mathrm{ng} /$ cells $/$ day & {$[11]$} \\
\hline$d_{c_{1}}$ & Decay rate of $c_{1}$ & $12.79-55 /$ day & {$[29,38]$} \\
\hline$d_{c_{2}}$ & Decay rate of $c_{2}$ & $2.5-4.632 /$ day & {$[29,38]$} \\
\hline$a_{12}$ & Effectiveness of $c_{2}$ inhibition of $c_{1}$ synthesis & $0.025 \mathrm{ng} / \mathrm{mL}$ & [29] \\
\hline$a_{22}$ & Effectiveness of $c_{2}$ inhibition of $c_{2}$ synthesis & $0.1 \mathrm{ng} / \mathrm{mL}$ & [29] \\
\hline$a_{p m}$ & Effectiveness of $c_{1}$ inhibition of $C_{m}$ proliferation & $3.162 \mathrm{ng} / \mathrm{mL}$ & {$[11,46]$} \\
\hline$a_{m b_{1}}$ & Effectiveness of $c_{1}$ inhibition of $C_{m}$ differentiation & $0.1 \mathrm{ng} / \mathrm{mL}$ & {$[11,47]$} \\
\hline$a_{01}$ & Half-saturation of $c_{1}$ to activate $M_{1}$ & $0.01 \mathrm{ng} / \mathrm{mL}$ & [29] \\
\hline$a_{02}$ & Half-saturation of $c_{2}$ to activate $M_{2}$ & $0.005 \mathrm{ng} / \mathrm{mL}$ & [29] \\
\hline$a_{p b}$ & Effectiveness of $c_{1}$ inhibition of $C_{b}$ proliferation & $10 \mathrm{ng} / \mathrm{mL}$ & {$[11,48]$} \\
\hline$a_{p m_{1}}$ & Constant enhancement of $c_{1}$ to $C_{m}$ proliferation & $13 \mathrm{ng} / \mathrm{mL}$ & {$[11,46]$} \\
\hline$k_{p m}$ & Proliferation rate of $C_{m}$ & $0.5 /$ day & [11] \\
\hline$d_{m}$ & Differentiation rate of $C_{m}$ & $1 /$ day & {$[11,30]$} \\
\hline$k_{p b}$ & Proliferation rate of $C_{b}$ & $0.2202 /$ day & {$[11,30]$} \\
\hline$d_{b}$ & Differentiation rate of $C_{b}$ & $0.15 /$ day & {$[11,30]$} \\
\hline$p_{c S}$ & Fibrocartilage synthesis rate & $3 \times 10^{-6} \mathrm{~g} /$ cells $/$ day & {$[11,30]$} \\
\hline$q_{c d_{1}}$ & Fibrocartilage degradation rate & $3 \times 10^{-6} \mathrm{~mL} /$ cells $/$ day & {$[11,30]$} \\
\hline$q_{c d_{2}}$ & Fibrocartilage degradation rate by osteoclasts & $0.2 \times 10^{-6} \mathrm{~mL} /$ cells $/$ day & {$[11,30]$} \\
\hline$p_{b s}$ & Bone tissue synthesis rate & $5 \times 10^{-8} \mathrm{~g} /$ cells/day & {$[11,30]$} \\
\hline$q_{b d}$ & Bone tissue degradation rate & $5 \times 10^{-8} \mathrm{~mL} /$ cells $/$ day & [30] \\
\hline$K_{l b}$ & Carrying capacity of $C_{b}$ & $1 \times 10^{6}$ cells $/ \mathrm{mL}$ & {$[11,30]$} \\
\hline$K_{l m}$ & Carrying capacity of $C_{m}$ & $1 \times 10^{6}$ cells $/ \mathrm{mL}$ & {$[11,30]$} \\
\hline$D(0)$ & Density of necrotic cells & $1 \times 10^{6}-2 \times 10^{8}$ cells $/ \mathrm{mL}$ & {$[27,38,41]$} \\
\hline$C_{m}(0)$ & Initial MSCs density & 1000 cells $/ \mathrm{mL}$ & {$[11]$} \\
\hline$M_{0}(0)$ & Unactivated macrophage density & 4000 cell $/ \mathrm{mL}$ & [45] \\
\hline
\end{tabular}
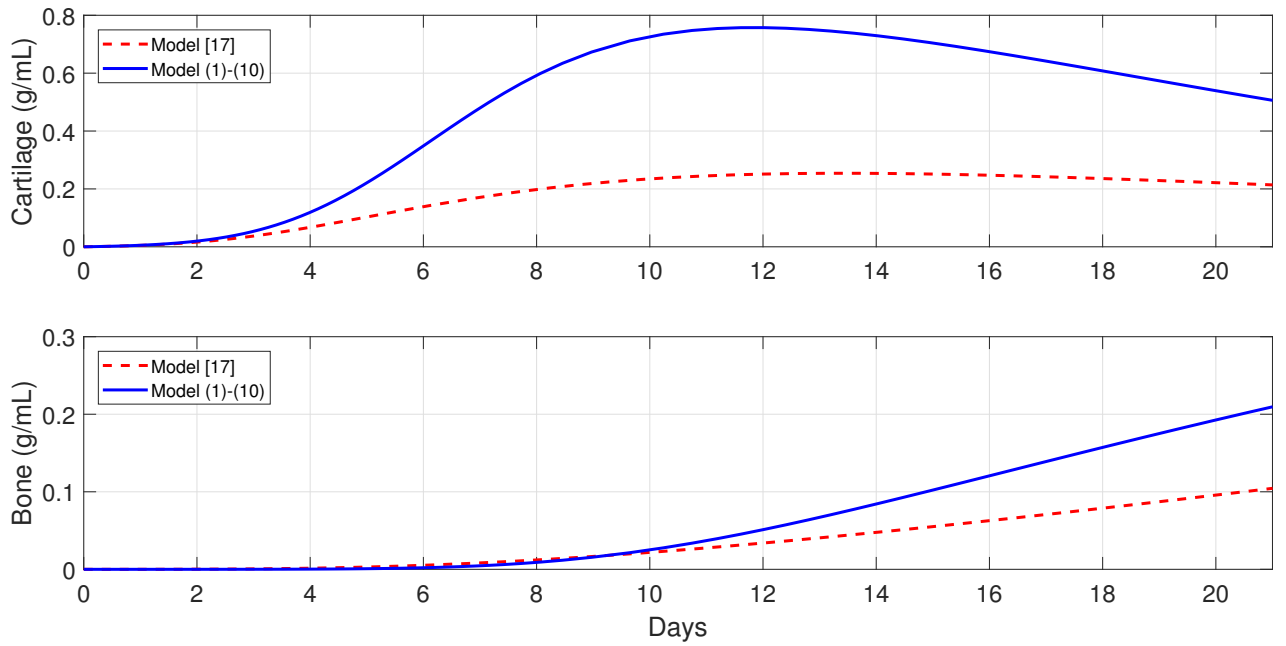

Figure 3. Comparison of tissues evolution in Model [11] and Model (1)-(10). 


\subsection{Different Outcomes of the Bone Fracture Healing Process}

Next, a set of numerical simulations is presented to support the theoretical results. Accordingly to the qualitative analysis of the model there are three equilibria: $E_{0}, E_{1}$, and $E_{2}$, where their stability conditions are determined by the tissue cells' proliferation and differentiation rates, $k_{p m}, k_{p b}, d_{m}$ and $d_{b}$, respectively. The following parameter values were used: $k_{p m}=0.5, d_{m}=1, k_{p b}=0.2202$, and $d_{b}=0.3$, to demonstrate the stability of $E_{0}$, since then $k_{p m}<d_{m}$ and $k_{p b}<d_{b}$. The stability of $E_{1}$ was demonstrated using the following parameter values: $d_{m}=1, k_{p m}=0.5, k_{p b}=0.2202$, and $d_{b}=0.15$, since then $k_{p m} \leq d_{m}$ and $k_{p b}>d_{b}$. Finally, the following parameter values were used: $k_{p m}=0.5$ and $d_{m}=0.1$, to demonstrate the stability of $E_{2}$, since then $k_{p m}>d_{m}$. Different time-periods are used in Figures 4-6 to better demonstrate the qualitative behavior of the system under different stability conditions.

Figure 4 shows the qualitative behavior of $E_{1}$ for the macrophages, debris, TNF- $\alpha$, and IL-10 densities, with the inflammation being resolved in about 40 days. The top-left plot of Figure 4 shows the temporal evolution of $M_{0}$ (dashed lines), $M_{1}$ (dotted lines), and $M_{2}$ (solid lines). It can be observed that $M_{1}$ first peaks to its maximum value, which is then followed by $M_{2}$. Similar sequences of transitions of first $M_{1}$, and then $M_{2}$, are commonly observed in normal tissue healing conditions [2,39].
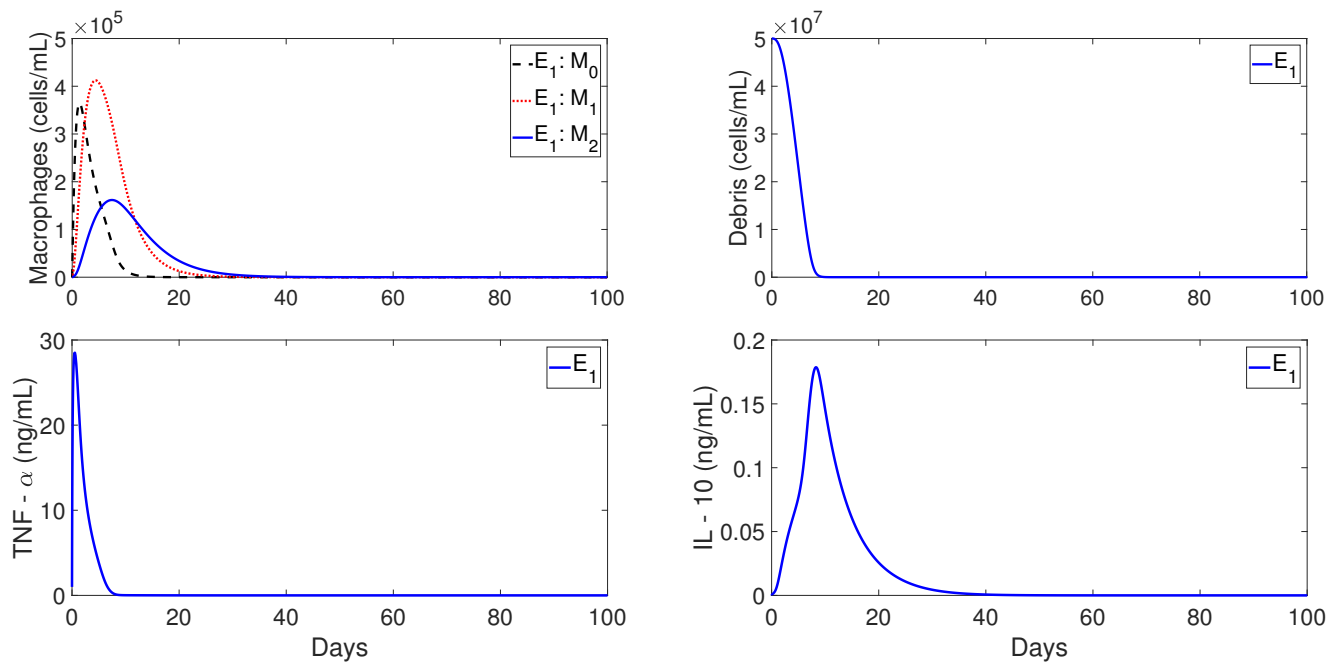

Figure 4. Cellular and molecular evolution of the resolution of the inflammation in normal conditions.
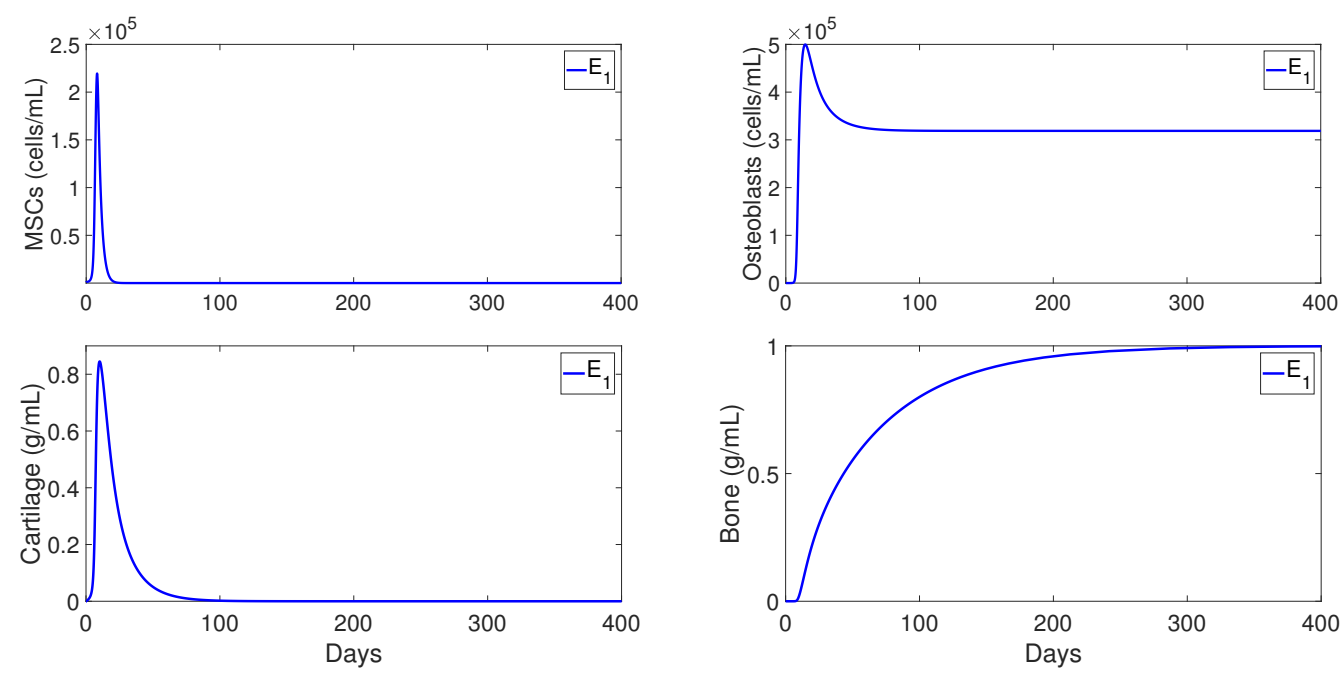

Figure 5. Cellular and molecular evolution of the repair process in a successful fracture healing. 
Figure 5 shows the qualitative behaviors of $E_{1}$ for the MSC, osteoblasts, cartilage, and bone densities. Here, the MSC density decayed to zero over time, while the osteoblasts maintained a constant density below their carrying capacity $K_{l b}=1 \times 10^{6}$. In addition, the bottom plots of Figure 5 shows that the cartilage was eventually degraded by the osteoclasts and the bone achieved its maximum density of $1 \mathrm{ng} / \mathrm{mL}$. Therefore, $E_{1}$ exhibits the temporal progression of a successful bone fracture healing.
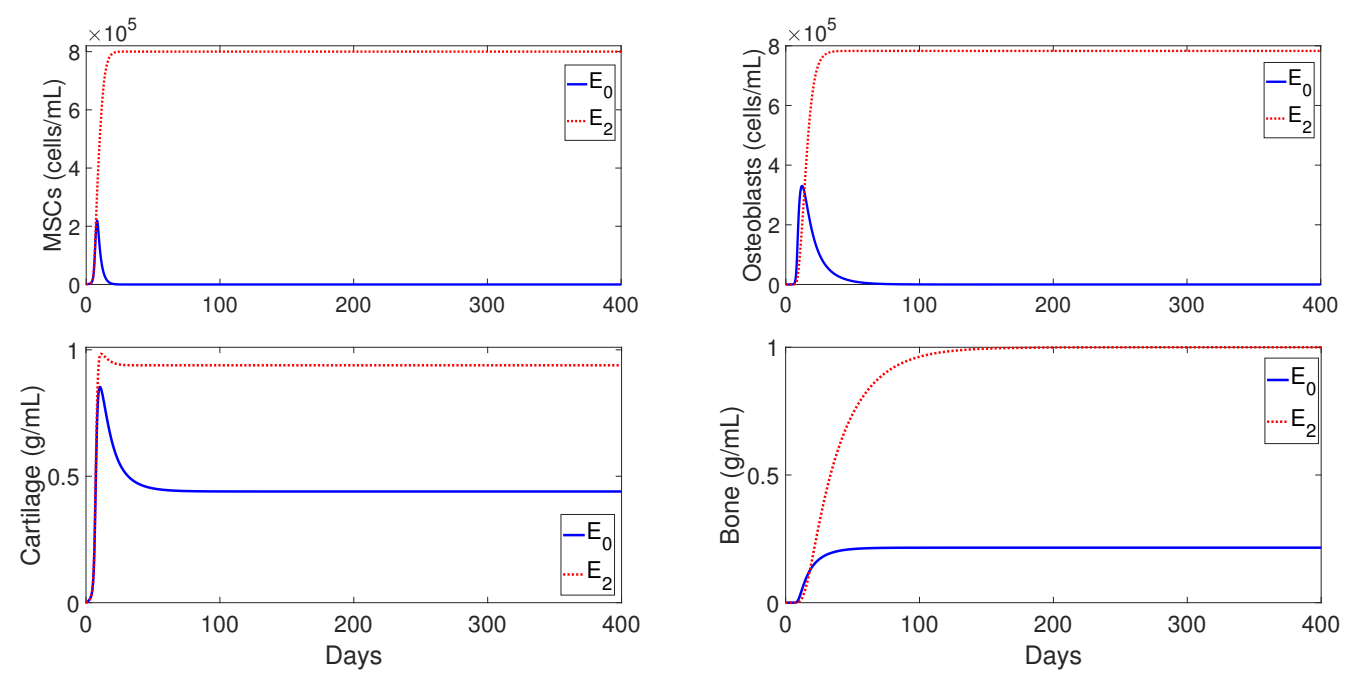

Figure 6. Cellular and molecular evolution of the repair process in a nonunion fracture healing.

Figure 6 shows the qualitative evolution for the MSC, osteoblasts, cartilage, and bone densities for $E_{0}$ (solid lines) and $E_{2}$ (dotted lines). Since the temporal evolution of macrophages, debris, and cytokines densities in $E_{0}$ and $E_{2}$ are similar to those for $E_{1}$ showed in Figure 4, they are omitted here. It can be observed, in Figure 6, that the two cellular densities in $E_{0}$, MSC, and osteoblasts, decayed to zero over time, with the osteoclasts failing to degrade the cartilage; this results in nonunion. Mathematically, this case occurs when osteoblasts proliferate at a rate lower than their differentiation rate, i.e., $k_{p b}<d_{b}$. In practice, this scenario is commonly observed in advanced-age patients whose MSC and osteoblast cells decrease their capability to proliferate and differentiate [3]. On the other hand, the two cells and the two tissues in $E_{2}$ remain at positive constant values (Figure 6), but the final fracture healing outcome is still a nonunion. Here, the osteoclasts again fail to degrade the cartilage [3], even though the bone has achieved its maximum density of $1 \mathrm{ng} / \mathrm{mL}$. Therefore, in this case, migration of osteoclasts must be enhanced through surgical interventions, in order to achieve a successful bone repair [30].

\subsection{Importance of Macrophages during the Bone Fracture Healing Process}

In this section, the mathematical model is used to investigate the effects of macrophages during the inflammatory and repair phases of the bone fracture healing process. The major contribution of macrophages to fracture healing is through the delivery of pro- and anti-inflammatory cytokines at the repair site. Therefore, the values of the parameters $k_{i}$, representing the secretion rates of $c_{i}$ by $M_{i}$, $i=1,2$, are varied in the numerical simulations, as compared to their base values from Table 3 .

Figure 7 shows that macrophages have a drastic effect on the short-term tissue dynamics during the healing process. In the presence of $M_{1}$ and $M_{2}$, fibrocartilage formation more than doubles in about 1 week, while woven bone experiences an additional steady increase during the same period and beyond. The simulations presented in Figure 8 demonstrate the individual effects of the different phenotypes of macrophages, and show that the alternatively activated macrophages $M_{2}$ have a more dominant contribution to the tissue production, as compared to the classically activated macrophages $M_{1}$. 

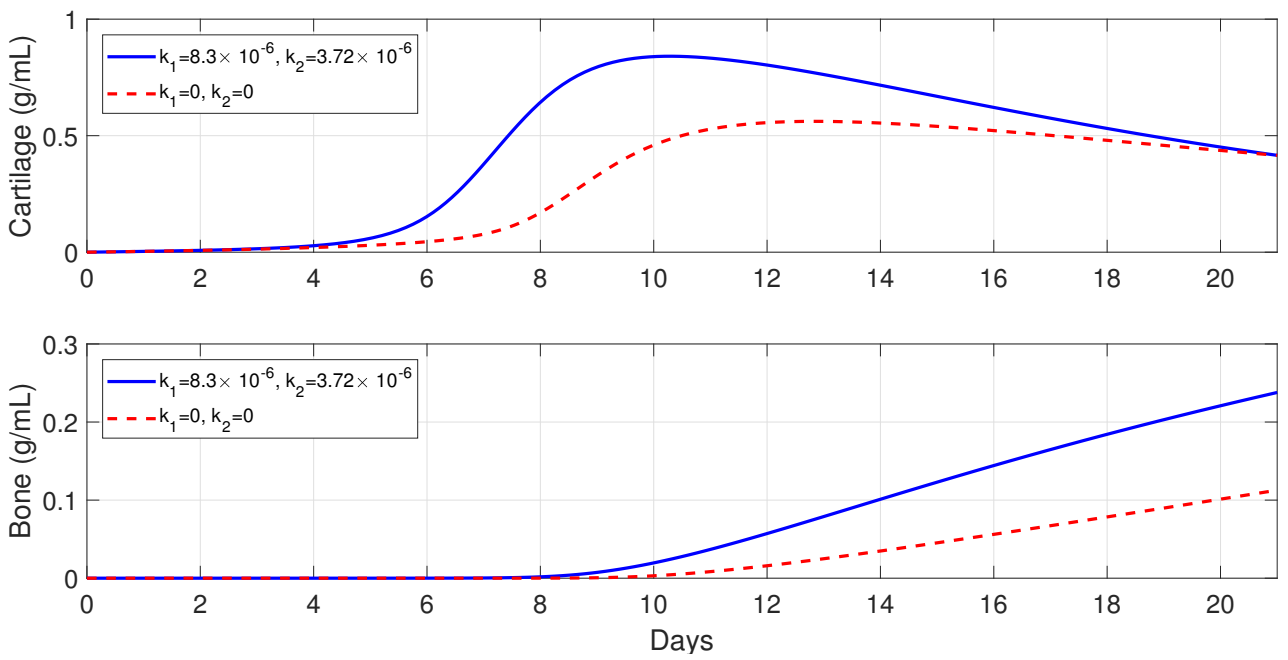

Figure 7. Tissue evolution when macrophages contribute to the healing process (solid line), $k_{1}, k_{2} \neq 0$, and when they do not contribute to the healing process (dashed line), $k_{1}=k_{2}=0$.
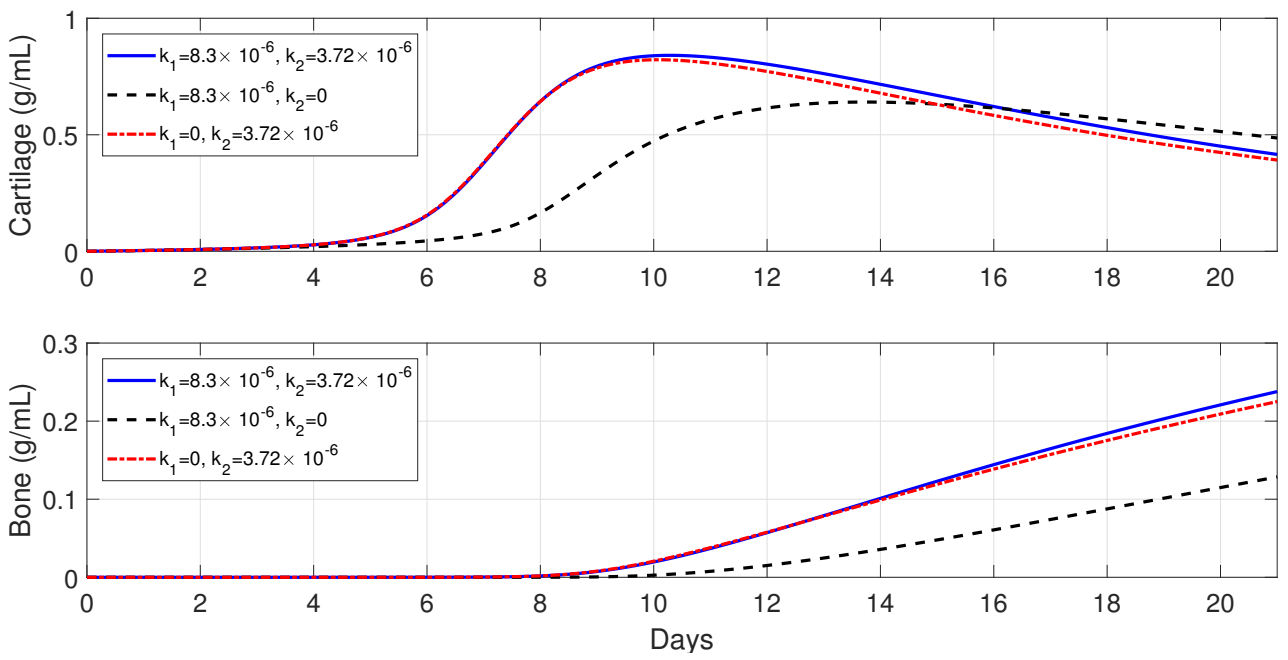

Figure 8. Tissue evolution when the alternatively activated macrophages, $M_{2}$, do not contribute to the healing process (dashed line), $k_{2}=0$, and when the classically activated macrophages, $M_{1}$, do not contribute to the healing process (dotted line), $k_{1}=0$.

\subsection{Evolution of the Healing Process for Different Types of Fractures}

In this section, the model is used to monitor the evolution of a successful repair (Table 3) for different types of fractures. In healthy individuals, simple, moderate, and severe fractures are correlated with the debris densities $[49,50]$. Therefore, the initial debris concentration is set to $D(0)=3 \times 10^{5}$, $D(0)=5 \times 10^{7}$, and $D(0)=2 \times 10^{8}$, for a simple, moderate, and severe fracture, respectively.

Figure 9 shows that the tissue production is a slow process for a simple fracture, since the cartilage and bone densities are less than the corresponding tissue densities for moderate and severe fractures. A slow healing process is commonly observed in micro-crack healing [49]. Furthermore, there is less cartilage formation over time in simple fractures [50]. For a moderate fracture, the maximal production of cartilage is observed around 10 days, followed by a significant degradation, while the bone tissue production occurs after the first week. For a severe fracture, Figure 9 shows that there is a delay in the two tissues' production, compared with those given by moderate fractures, with the peak of the cartilage and bone production observed at around day 16. 

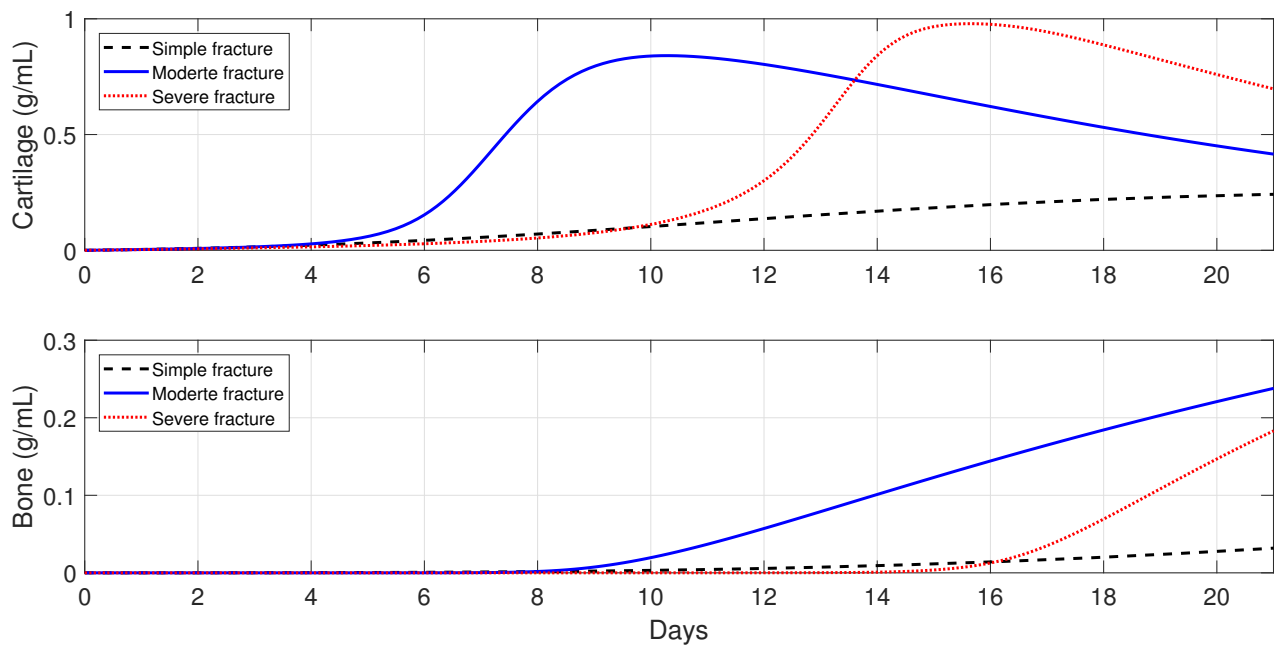

Figure 9. Tissue evolution of a successful repair for different types of fractures.

\subsection{Immune-Modulation Therapeutic Treatments of Bone Fractures}

The administration of anti-inflammatory drugs and the injection and/or transplantation of MSC and macrophages are two of the clinical trials that have been implemented in orthopedics to stimulate and accelerate bone fracture healing [2,23]. In this section, Model (1)-(10) is used to explore these possible therapeutic treatments to accelerate the healing of a broken bone, under normal and pathological conditions such as severe fractures, advanced age, and senile osteoporosis [3].

\subsubsection{Administration of Anti-Inflammatory Drugs at the Beginning of the Healing Process}

Treatments based on anti-inflammatory cytokines, such as the cytokine-specific agents that block the pro-inflammatory cytokines productions, have exhibited promising clinical results and have led to intense orthopedic research activities $[1,2,17,23,33,51-53]$. In this section, a set of numerical simulations is presented to investigate the effect of the administrations of anti-inflammatory cytokines at the beginning of the healing process in healthy individuals and also in immune-compromised patients. In each case of the numerical simulations, $c_{2}(0)=0,10$, and $100 \mathrm{ng} / \mathrm{mL}$.

In healthy individuals, the administration of anti-inflammatory drugs is implemented for a simple fracture and also for two moderate fractures with different debris concentrations: $D(0)=3 \times 10^{5}$, $D(0)=2 \times 10^{7}$, and $D(0)=5 \times 10^{7}$.

Figure 10 shows that the administration of $c_{2}$ in the simple fracture slows down both the cartilage and bone productions. Figures 11 and 12 show that the administration of $c_{2}$ in the moderate fractures improves the tissue evolution, but in a dose-dependent manner. On one hand, when $D(0)=2 \times 10^{7}$, the administration of $c_{2}$ has either a positive or negative effect on the two tissue productions. The administration of $10 \mathrm{ng} / \mathrm{mL}$ of $c_{2}$ enhances the early production of cartilage and increases the bone synthesis, while the administration of $100 \mathrm{ng} / \mathrm{mL}$ of $c_{2}$ results in the opposite effect. On the other hand, when $D(0)=5 \times 10^{7}$, the administration of $c_{2}$ enhances the earlier cartilage production and improves the synthesis of the bone for both concentrations, with $10 \mathrm{ng} / \mathrm{mL}$ being the optimal of the two doses. 

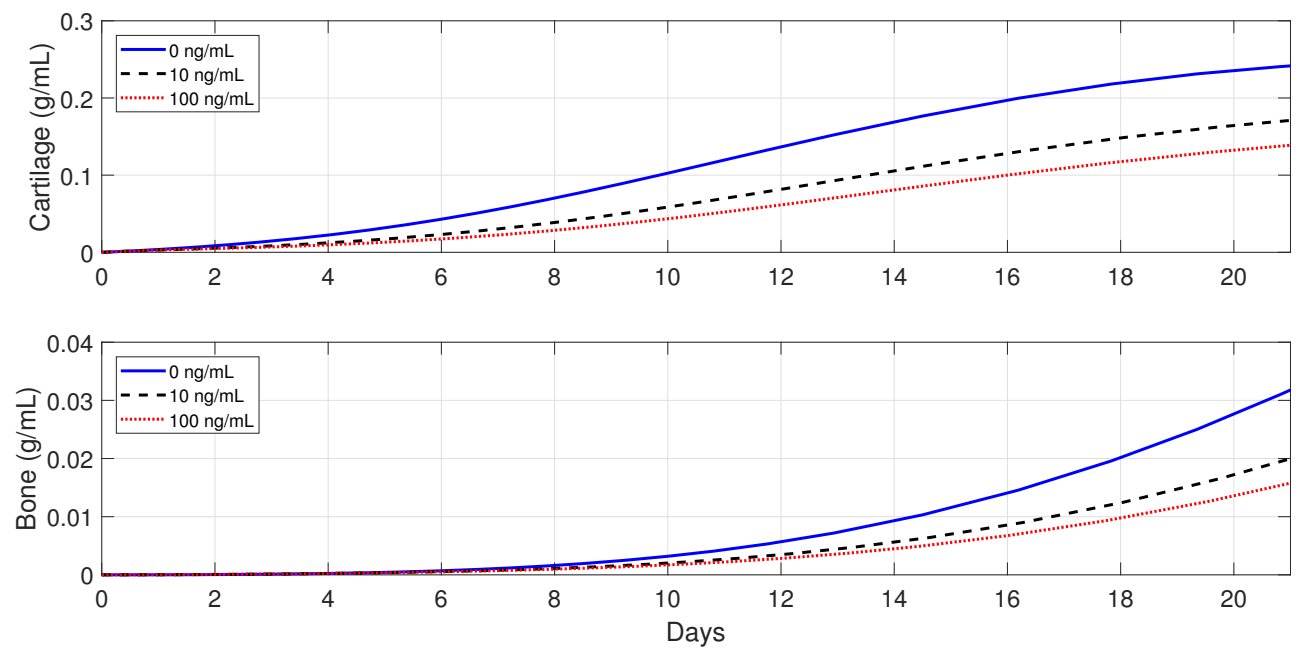

Figure 10. Tissue evolution in a simple fracture under different initial anti-inflammatory cytokines concentrations, $D(0)=3 \times 10^{5}$.
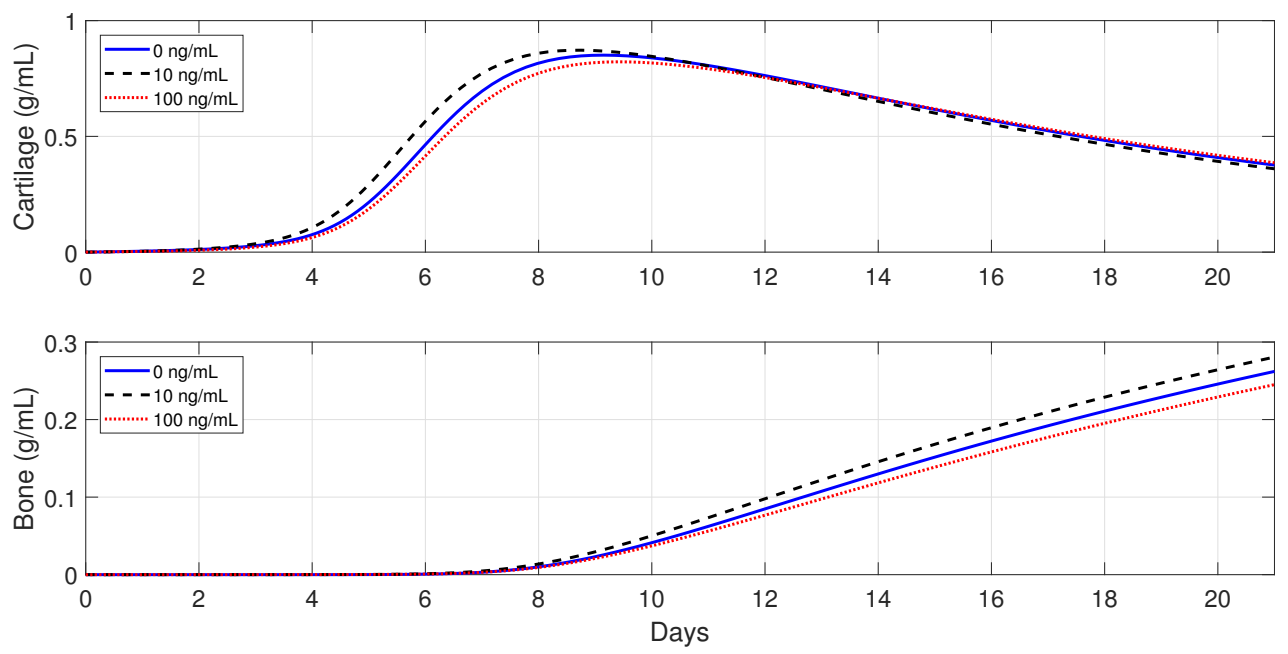

Figure 11. Tissue evolution in a moderate fracture under different initial anti-inflammatory cytokines concentrations, $D(0)=2 \times 10^{7}$.

Next, the model is used to implement the administration of anti-inflammatory drugs under different pathological conditions. First, severe fractures in immune-compromised individuals are simulated by using the following parameter values: $D(0)=2 \times 10^{8}$ and $k_{\max }=0.0015$, since, in the fractures of such individuals, there is an increase in the accumulation of debris [50] and a decrease in the macrophage migration rate [54]. Second, the following parameter values are used: $k_{e_{1}}=k_{e_{2}}=3$ and $k_{1}=9 \times 10^{-6}$, to simulate bone fracture healing in aging individuals, since, in this case, the macrophage phagocytic rate decreases and there is an increase of pro-inflammatory cytokine synthesis by $M_{1}[3,25]$. Finally, $c_{1}(0)=100, k_{p m}=0.2, d_{m}=0.5, k_{p b}=0.16$, and $d_{b}=0.15$ are used to simulate the healing process for an senil osteoporotic fracture, since, in this case, a high level of pro-inflammatory cytokines is observed and the MSC and osteoblast functions decrease [3]. 

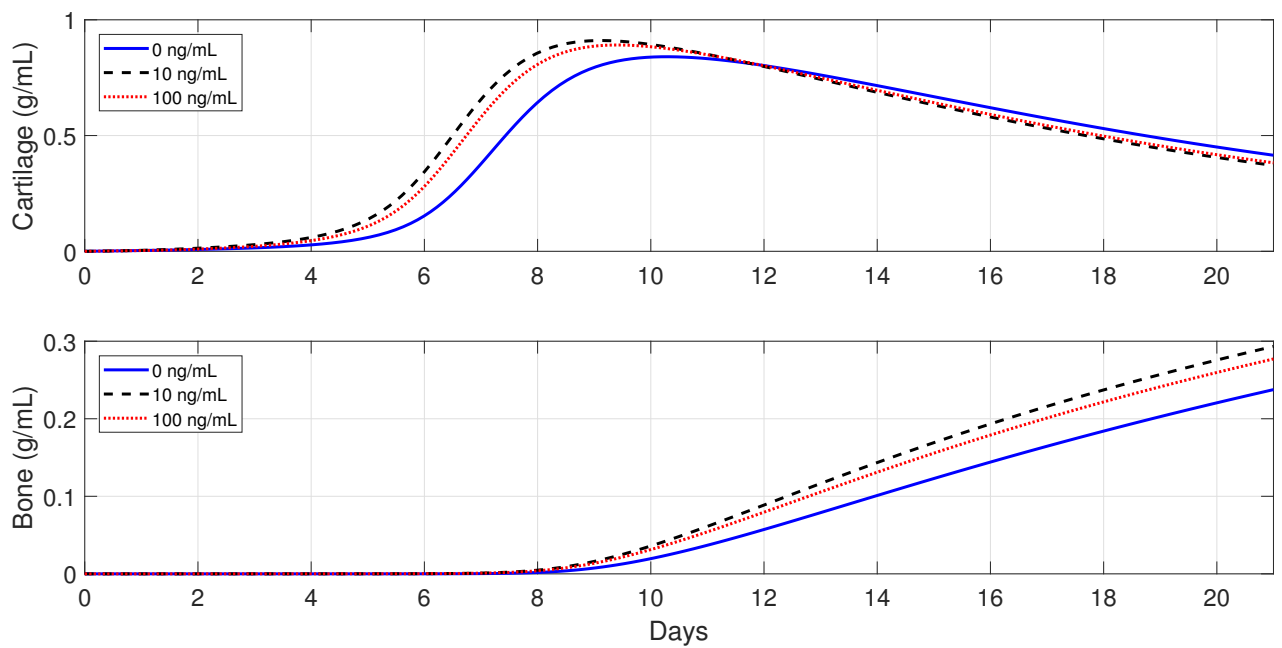

Figure 12. Tissue evolution in a moderate fracture under different initial anti-inflammatory cytokine concentrations, $D(0)=5 \times 10^{7}$.

Figures 13-15 show that the administration of anti-inflammatory cytokines under the above three different pathological conditions always improve tissue productions, where the optimal dose of $c_{2}$, for both the advanced-age individuals and senile osteoporotic fractures, is $10 \mathrm{ng} / \mathrm{mL}$.
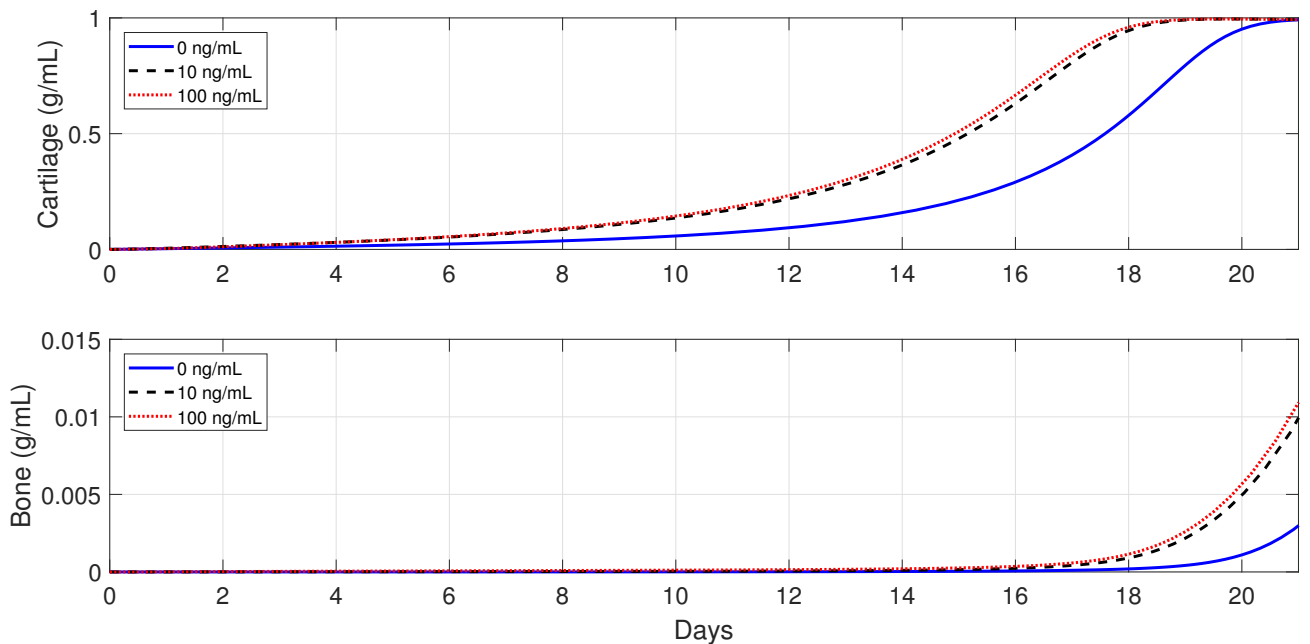

Figure 13. Tissue evolution in a severe fracture under different initial anti-inflammatory cytokines concentrations. 

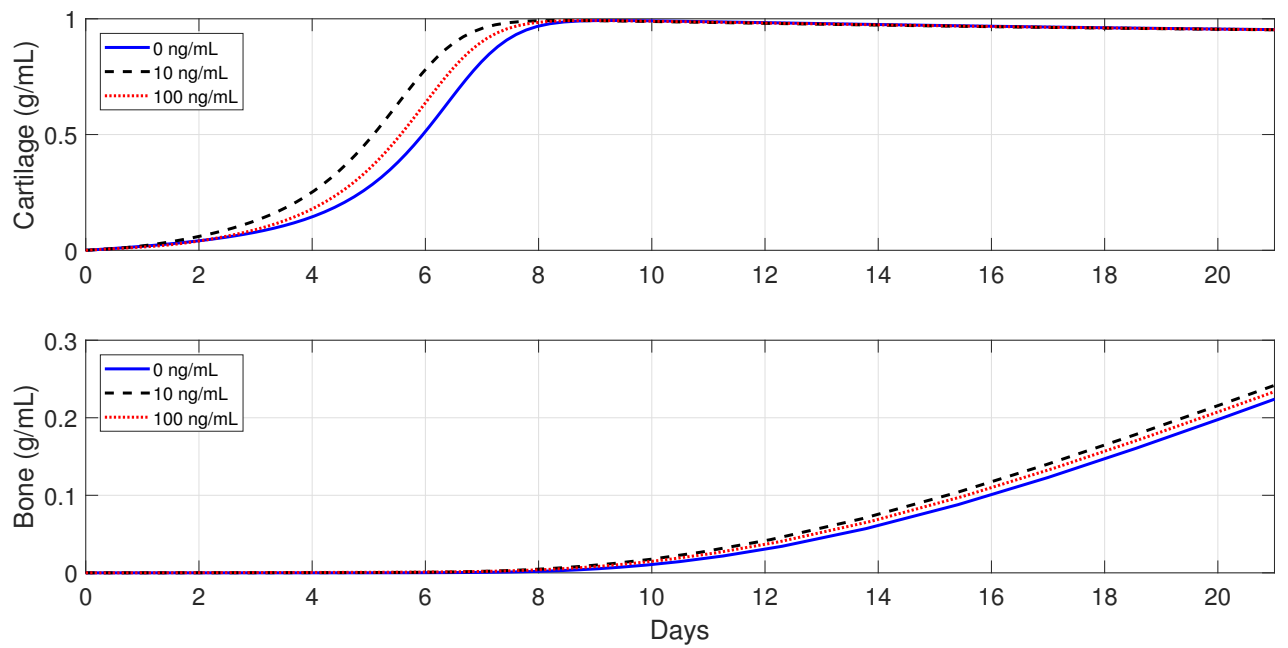

Figure 14. Tissue evolution in an advanced age fracture under different initial anti-inflammatory cytokines concentrations.
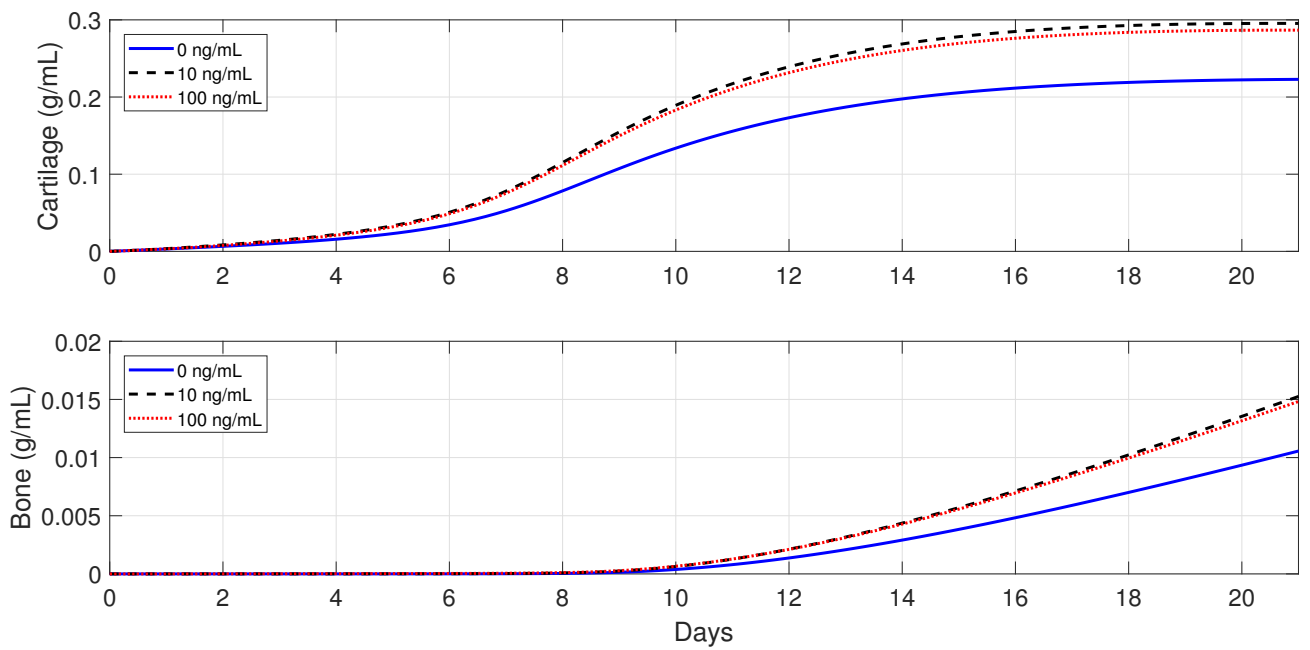

Figure 15. Tissue evolution in a senile osteoporotic fracture under different initial anti-inflammatory cytokines concentrations.

\subsubsection{Cellular Therapeutic Interventions under Immune-Compromised Conditions}

Additions of MSC to the injury site through injection and/or transplantation have been used in practice to stimulate and augment bone fracture healing [2]. Another cellular intervention is scaffold implants, where undifferentiated macrophages and MSCs are co-cultured together, and cytokines are slowly released to stimulate $M_{2}$ activation [3]. The parameter values used in the numerical simulations that explore these possible therapeutic treatments are the same as in Section 6.5.1.

For severe fractures with immune-compromised conditions, the use of scaffold implants is simulated through a fast $M_{2}$ activation (i.e., $k_{02}=0.3$ and $k_{12}=0.075$ ), and also an increase in the $C_{m}$ and $M_{0}$ densities (i.e., $M_{0}(0)=5000$ and $C_{m}(0)=5000$ ). For fractures in aging individuals and individuals with senile osteoporotic fractures, the MSC injection and the fast $M_{2}$ activation are simulated by setting $C_{m}(0)=5000, k_{02}=0.3$, and $k_{12}=0.075$.

Figures 16-18 show that the two cellular interventions increase both tissue productions. Furthermore, those interventions result in larger improvements in severe and senil osteoporotic fractures, when compared to fractures in aging individuals. 

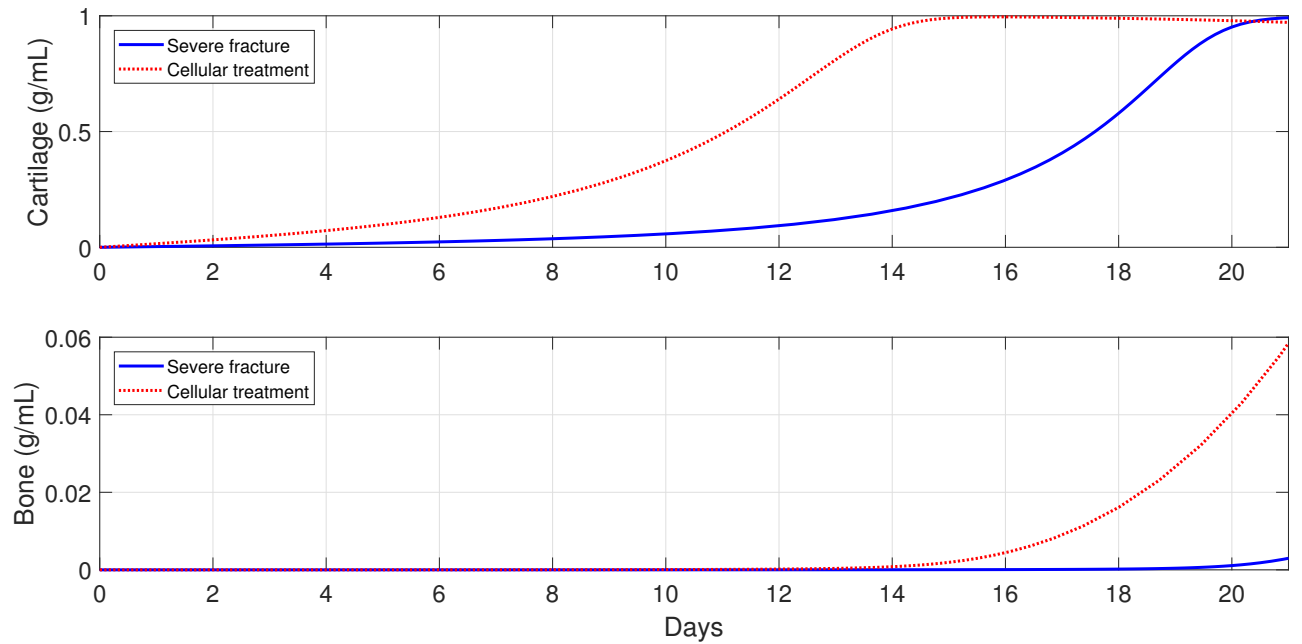

Figure 16. Tissue evolution in a severe fracture without therapeutic innervation (solid line) and with $M_{0}(0)$ and $C_{m}(0)$ transplantation (dotted line).
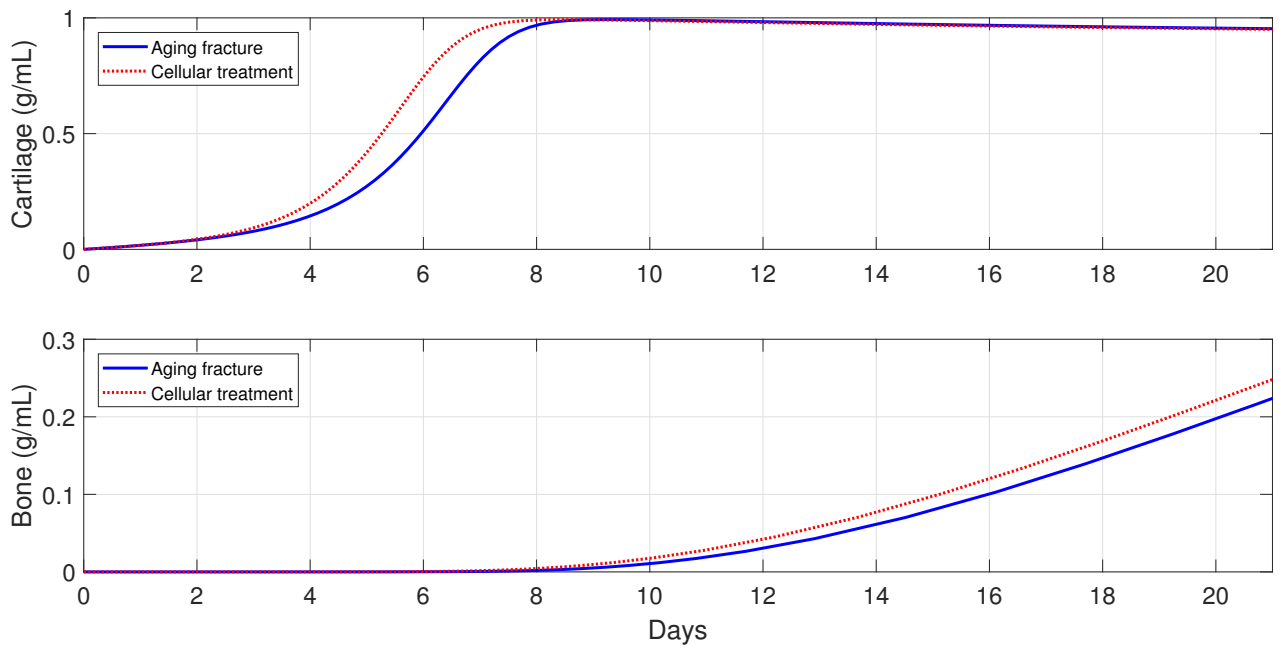

Figure 17. Tissue evolution in an aging fracture without therapeutic innervation (solid line) and with MSCs injection (dotted line).
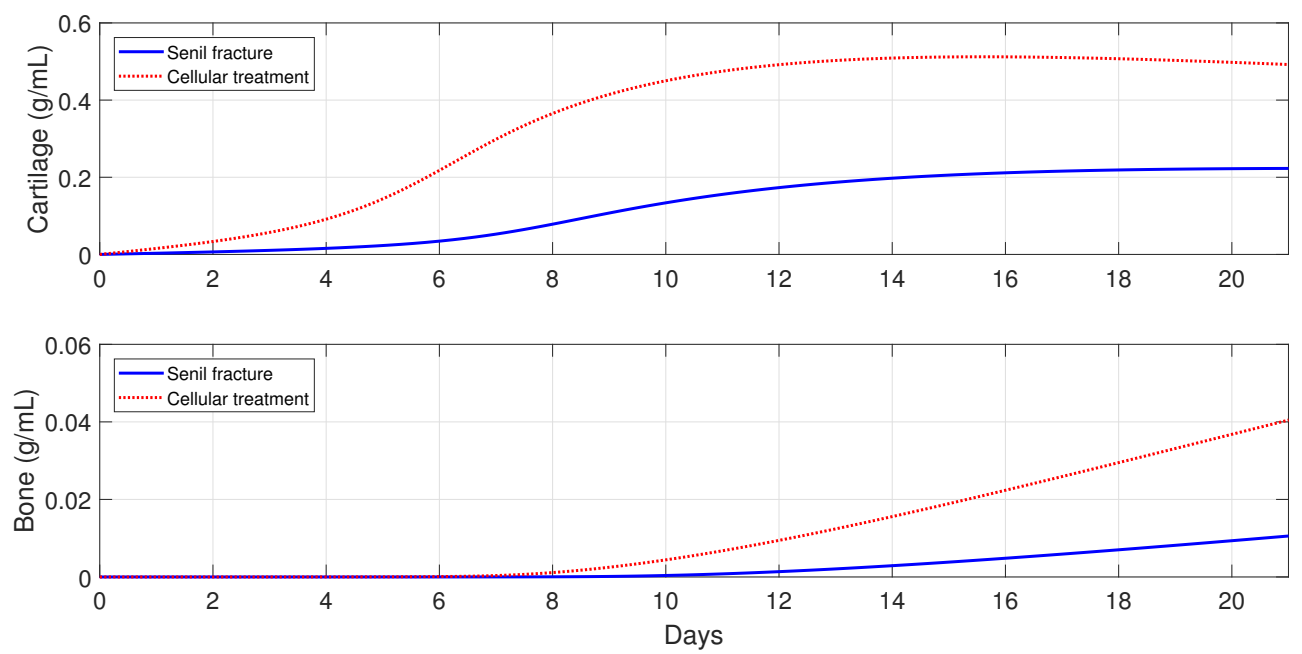

Figure 18. Tissue evolution in a senile osteoporotic fracture without therapeutic innervation (solid line) and with MSCs injection (dotted line). 


\section{Discussion and Conclusions}

A new mathematical model was introduced to mathematically and numerically study the macrophage-mediated inflammation involved in the early stages of the bone fracture healing process: The inflammatory and repair phases. Classically and alternatively activated macrophages were incorporated in the model, to study their capabilities to modulate and resolve the inflammation. The model also included the macrophage abilities to regulate the tissue cellular functions through the delivery of pro- and anti-inflammatory cytokines. The resolution of the inflammation was assumed to be initiated with the activation of the macrophages into their classical phenotype. The classically activated macrophages deliver pro-inflammatory cytokines, such as TNF- $\alpha$, as they engulf debris. Then, the alternatively activated macrophages and the MSC modulate the inflammation by releasing anti-inflammatory cytokines, such as IL-10. Finally, the classically activated macrophages remove the remaining debris. The model also incorporated different engulfing rates of activated macrophages, saturation rates of phagocytes, and the maximal density of macrophages at the injury site, thus allowing a better understanding of the interplay between macrophages and tissue cells during the bone fracture healing process.

The mathematical analysis revealed that there are three feasible fracture healing outcomes. Two of the outcomes represent a nonunion healing: One is the case when the cells deactivate or die out before the healing process finishes up, and the other is the case when the tissue cells remain constant but the osteoclasts fail to completely remove the cartilage. The third outcome represents a successful healing, where the osteoblasts and osteoclasts are constantly producing and removing the woven bone. The stability conditions of each outcome can be used to biologically explain why the fracture healing fails, as well as to design therapeutic interventions to stimulate or accelerate the healing process.

The new model was used to study the importance of macrophages during the early stages of tissue production. It revealed that macrophages significantly improve the tissue production, with alternatively activated macrophages having the main effect on the process. The flexibility of the model also allowed us to perform a variety of different types of numerical simulations quickly and cost effectively. It was used to monitor the progression of the healing of a broken bone and to predict its final outcome. In particular, the administration of anti-inflammatory drugs to improve the bone fracture healing process was numerically simulated. It was found that the administration of anti-inflammatory cytokines fails to accelerate the healing process in simple fractures, while it accelerates the healing process in moderate fractures, depending on the cytokine concentrations, and always improves the healing process in severe fractures. Such results have been also clinically observed, when corticosteroids and nonsteroidal anti-inflammatory drugs (NSAIDs) are administered in bone fractures [23]. Therefore, based on the model findings, the concentration of debris must be carefully considered when administering anti-inflammatory drugs to enhance the fracture healing process [50]. The model was also used to explore other potential cellular therapeutic approaches, such as MSC injection and transplantation. It was found that such treatments can also improve the healing time of a broken bone, especially in immune-compromised patients. The model can also be easily adapted to other therapeutic approaches, such as the administration of different anti-inflammatory drugs, suggesting a variety of possible ways to guide clinical experiments and bone tissue engineering strategies.

Future research directions include modifications of the model by incorporating additional molecular and cellular interactions and processes during the inflammatory and repair phases of fracture healing, such as macrophage migration and MSC differentiation due to cytokine stimulus. Another research direction is the incorporation of the bone remodeling phase of the healing process, which begins at the end of the repair phase and continues long after fracture union. There are different factors that affect the bone remodeling, including other bone cells, such as osteoclasts, osteocytes, progenitor cells, and other sources of cytokines $[30,32,43]$. This presents a challenging new research direction in the pursuit to better understand the bone fracture healing process and the development of new treatment strategies. 
Author Contributions: All authors contributed equally to the work reported.

Funding: This research was funded by Consejo Nacional de Ciencia y Tecnología grant No. Beca 383167.

Acknowledgments: The first author, I.T., was supported by the Mexico's CONACyT and the University of Texas at Arlington.

Conflicts of Interest: The authors declare no conflict of interest. The founding sponsors had no role in the design of the study; in the collection, analyses, or interpretation of data; in the writing of the manuscript, and in the decision to publish the results.

\section{Appendix A}

The stability conditions of the equilibria of Model (1)-(10) are stated and proved below. The analysis is conducted using the Jacobian of the system at each equilibrium point and finding its corresponding eigenvalues [40,55].

Theorem A1. The $E_{0}=\left\{\left(0,0,0,0,0,0,0,0, m_{c_{0}}^{*}, m_{b_{0}}^{*}\right)\right.$ belongs to the set $B=\left\{\left(0,0,0,0,0,0,0,0, m_{c}, m_{b}\right)\right.$ : $\left.0 \leq m_{c} \leq p_{c s} / q_{c d_{1}}, 0 \leq m_{b} \leq p_{b s} / q_{b d}\right\}$, which is a local attractor set of the solution set given by System (1)-(10) if and only if $k_{p m} \leq d_{m}$ and $k_{p b} \leq b_{d}$.

Proof of Theorem A1. The right-hand side functions of System (1)-(10) are continuous and bounded, since all model variables and parameters are positive. Hence, for each initial condition of the system, there is a unique solution [55]. Then, as zero is a solution of the System (1)-(10), and by uniqueness of solution, all the solutions of the system with positive initial condition are positive [55].

Next, it will be proved that the hyperplane $A=\left\{\left(0,0,0,0,0,0,0,0, m_{c}, m_{b}\right): m_{c} \geq 0, m_{b} \geq 0\right\}$ is an attractor set of the solutions of the system (1)-(10). There are two cases to consider, based on the relation between the cell proliferation and differentiation rates.

First, let us examine the case when $k_{p m}<d_{m}$ and $k_{p m}<d_{b}$. The Jacobian matrix $J\left(E_{0}\right)$ is given by the following lower triangular block matrix

$$
J\left(E_{0}\right)=\left(\begin{array}{ccc}
J_{1}\left(E_{0}\right) & \mathbf{0} & \mathbf{0} \\
* & J_{2}\left(E_{0}\right) & \mathbf{0} \\
\mathbf{0} & * & J_{3}\left(E_{0}\right)
\end{array}\right),
$$

where

$$
\begin{gathered}
J_{1}\left(E_{0}\right)=\left(\begin{array}{cccc}
0 & 0 & 0 & 0 \\
k_{\max } & -d_{0} & 0 & 0 \\
0 & 0 & J_{11} & 0 \\
k_{0} & 0 & * & -d_{c_{1}}
\end{array}\right), \quad J_{2}\left(E_{0}\right)=\left(\begin{array}{ccc}
-d_{c_{2}} & k_{3} & 0 \\
0 & -d_{m}+k_{p m} & 0 \\
0 & d_{m} & -d_{b}+k_{p b}
\end{array}\right) \\
J_{11}=\left(\begin{array}{cc}
-d_{1}-k_{12} & k_{21} \\
k_{12} & -d_{2}-k_{21}
\end{array}\right), \quad J_{3}\left(E_{0}\right)=\left(\begin{array}{ll}
0 & 0 \\
0 & 0
\end{array}\right) .
\end{gathered}
$$

Therefore, the corresponding characteristic polynomial associated with $J\left(E_{1}\right)$ is given by the product of the characteristic polynomials associated with each submatrix [56]:

$$
p(\lambda)=\lambda^{3}\left(\lambda+d_{0}\right)\left(\lambda+d_{c_{1}}\right)\left(\lambda+d_{c_{2}}\right)\left(\lambda+d_{m}-k_{p m}\right)\left(\lambda+d_{b}-k_{p m}\right)\left(\lambda^{2}+a \lambda+b\right),
$$

where $a=d_{1}+d_{2}+k_{12}+k_{21}$ and $b=k_{12} d_{2}+k_{21} d_{1}+d_{1} d_{2}$. The polynomial factor of order two of $p(\lambda)$ has the following two roots: $\left(-a \pm \sqrt{a^{2}-4 b}\right) / 2$, which are negative since $a^{2}-4 b=\left(d_{1}-d_{2}+k_{12}-\right.$ $\left.k_{21}\right)^{2}+4 k_{12} k_{21}>0$ and $b>0$. Therefore, the eigenvalues of $J\left(E_{0}\right)$ are negative for the variables $M_{0}$, $M_{1}, M_{2}, c_{1}, c_{2}, C_{m}$, and $C_{b}$ and are equal to zero for $D, m_{c}$, and $m_{b}$. Since $D^{\prime}(t) \leq 0$ for all the variables in the system (1)-(10) and $\left(D^{*}, 0,0,0,0,0,0,0, m_{c}, m_{b}\right)$ with $D^{*} \neq 0$ is not an equilibrium point, then the solutions of the system (1)-(8) are attracted to the set $A=\left\{\left(0,0,0,0,0,0,0,0, m_{c}, m_{b}\right): m_{c} \geq 0, m_{b} \geq 0\right\}$. 
Equations (9) and (10) imply that $m_{c}^{\prime} \leq 0$ and $m_{b}^{\prime} \leq 0$ for all $m_{c}>p_{c s} / q_{c d_{1}}$ and $m_{b}>p_{b s} / q_{b d}$. Therefore, the set $B$ is a local attractor set of $A$ [55].

Next, let us consider the case when $k_{p m}=d_{m}$ and $d_{b}=k_{p b}$. Here, the eigenvalues of $J\left(E_{0}\right)$ are the same as above except those associated with $C_{m}$ and $C_{b}$, which are equal to zero. Therefore, in this case, by considering the second order approximations of the right hand sides of Equations (7) and (8), instead of just the first order approximations, and using similar arguments as above, proves that the set $B$ is a local attractor set of $A$.

Theorem A2. The equilibrium $E_{1}=\left(0,0,0,0,0,0,0, K_{l b}\left(1-d_{b} / k_{p b}\right), 0, p_{b s} / q_{b d}\right)$ is locally stable if and only if $d_{m} \geq k_{p m}$ and $k_{p b}>d_{b}$.

Proof of Theorem A2. The Jacobian matrix corresponding to the point $E_{1}$ is given by the following lower triangular block matrix

$$
J\left(E_{1}\right)=\left(\begin{array}{ccc}
J_{1}\left(E_{1}\right) & \mathbf{0} & \mathbf{0} \\
* & J_{2}\left(E_{1}\right) & \mathbf{0} \\
\mathbf{0} & * & J_{3}\left(E_{1}\right)
\end{array}\right)
$$

where $J_{1}\left(E_{1}\right)$ has the same expression as $J_{1}\left(E_{0}\right)$, defined in Theorem $\mathrm{A} 1$, and

$$
J_{2}\left(E_{1}\right)=\left(\begin{array}{ccc}
-d_{c_{2}} & k_{3} & 0 \\
0 & -d_{m}+k_{p m} & 0 \\
0 & d_{m} & d_{b}-k_{p b}
\end{array}\right), \quad J_{3}\left(E_{1}\right)=\left(\begin{array}{cc}
-q_{c d_{2}} K_{l b}\left(1-\frac{d_{b}}{k_{p b}}\right) & 0 \\
0 & -q_{b d} K_{l b}\left(1-\frac{d_{b}}{k_{p b}}\right)
\end{array}\right) .
$$

Since $d_{m}-k_{p m} \geq 0$ and $k_{p b}>d_{b}$, and all of the eigenvalues of $J_{1}\left(E_{0}\right)$ are non-positive values, then the eigenvalues of $J\left(E_{1}\right)$ are negative, except the eigenvalues associated with $D$ and $C_{m}$ when $k_{p m}=d_{m}$, which are equal to zero. Therefore, $E_{1}$ is a locally stable node, since $D^{\prime} \leq 0$ for all the variables of the system (1)-(10) and $C_{m}^{\prime} \leq 0$ when $k_{p m}=d_{m}$.

Theorem A3. The equilibrium $E_{2}=\left(0,0,0,0,0, c_{2}^{*}, C_{m}^{*}, C_{b}^{*}, m_{c}^{*}, p_{b s} / q_{b d}\right)$ is locally stable if and only if $k_{p m}>d_{m}$, where $C_{m}^{*}=K_{l m}\left(1-d_{m} / k_{p m}\right), C_{b}^{*}=K_{l b}\left(k_{p b}-d_{b}+\sqrt{\left(k_{p b}-d_{b}\right)^{2}+4 k_{p b} d_{m} C_{m}^{*} / K_{l b}}\right) / 2 k_{p b}$, $c_{2}^{*}=a_{22}\left(-1+\sqrt{1+4 k_{3} C_{m}^{*} / a_{22} d_{c_{2}}}\right) / 2$, and $m_{c}^{*}=p_{c s} C_{m}^{*} /\left(q_{c d 1} C_{m}^{*}+q_{c d 2} C_{b}^{*}\right)$.

Proof of Theorem A3. The Jacobian matrix corresponding to the point $E_{2}$ is given by the following lower triangular block matrix

$$
J\left(E_{2}\right)=\left(\begin{array}{ccc}
J_{1}\left(E_{2}\right) & \mathbf{0} & \mathbf{0} \\
* & J_{2}\left(E_{2}\right) & \mathbf{0} \\
\mathbf{0} & * & J_{3}\left(E_{2}\right)
\end{array}\right)
$$

where

$$
\begin{aligned}
J_{1}\left(E_{2}\right)= & \left(\begin{array}{cccc}
0 & 0 & \mathbf{0} & 0 \\
k_{\max } & -d_{0}-G_{2}^{*} & \mathbf{0} & 0 \\
\mathbf{0} & * & J_{11} & \mathbf{0} \\
k_{0} H_{1}^{*} & 0 & * & -d_{c_{1}}
\end{array}\right), \quad J_{3}\left(E_{2}\right)=\left(\begin{array}{cc}
-q_{c d_{1}} C_{m}^{*}-q_{c d_{2}} C_{b}^{*} & 0 \\
0 & -q_{b d} C_{b}^{*}
\end{array}\right), \\
& J_{2}\left(E_{2}\right)=\left(\begin{array}{ccc}
-d_{c_{2}}\left(1+\frac{c_{2}^{*}}{a_{22}+c_{2}^{*}}\right) & k_{3} H_{2}^{*} & 0 \\
0 & d_{m}-k_{m b} & 0 \\
0 & d_{m} & -\sqrt{\left(d_{b}-k_{p b}\right)^{2}+4 \frac{k_{b p} d_{m} C_{m}^{*}}{K_{l b}}}
\end{array}\right),
\end{aligned}
$$


$G_{2}^{*}=\frac{c_{2}^{*} k_{02}}{a_{02}+c_{2}^{*}}, H_{1}^{*}=\frac{a_{12}}{a_{12}+c_{2}^{*}}, H_{2}^{*}=\frac{a_{22}}{a_{22}+c_{2}^{*}}$ and $J_{11}$ is defined as in Theorem A1. Since all of the eigenvalues of $J_{11}$ are negative (Theorem A1) and $k_{p m}>d_{m}$, and all equilibrium variables and parameter values are positive, then all the eigenvalues of $J_{1}\left(E_{2}\right), J_{2}\left(E_{2}\right), J_{3}\left(E_{2}\right)$ are negative, except for the eigenvalue associated to $D$ which is equal to zero. Therefore, since $D^{\prime} \leq 0$ for all the variable system, then $E_{2}$ is locally stable.

\section{References}

1. Schmidt-Bleek, K.; Kwee, B.J.; Mooney, D.J.; Duda, G.N. Boon and bane of inflammation in bone tissue regeneration and its link with angiogenesis. Tissue Eng. Part B Rev. 2015, 21, 354-364. [CrossRef] [PubMed]

2. Kovach, T.K.; Dighe, A.S.; Lobo, P.I.; Cui, Q. Interactions between MSCs and immune cells: Implications for bone healing. J. Immunol. Res. 2015, 2015, 752510. [CrossRef] [PubMed]

3. Loi, F.; Córdova, L.A.; Pajarinen, J.; Lin, T.; Yao, Z.; Goodman, S.B. Inflammation, fracture and bone repair. Bone 2016, 86, 119-130. [CrossRef] [PubMed]

4. Pisani, P.; Renna, M.D.; Conversano, F.; Casciaro, E.; Di Paola, M.; Quarta, E.; Muratore, M.; Casciaro, S. Major osteoporotic fragility fractures: Risk factor updates and societal impact. World J. Orthop. 2016, 7, 171. [CrossRef] [PubMed]

5. Teng, G.G. Mortality and osteoporotic fractures: Is the link causal, and is it modifiable? Clin. Exp. Rheumatol. 2008, 26, 125.

6. Carlier, A.; Geris, L.; Lammens, J.; Van Oosterwyck, H. Bringing computational models of bone regeneration to the clinic. Wiley Interdisciplin. Rev. Syst. Biol. Med. 2015, 7, 183-194. [CrossRef] [PubMed]

7. Huber-Lang, M.; Kovtun, A.; Ignatius, A. The role of complement in trauma and fracture healing. Semin. Immunol. 2013, 25, 73-78. [CrossRef]

8. Foundation, I.O. Osteoporosis Facts and Statistics; Data and Publication, International Osteoporosis Foundation: Nyon, Switzerland, 2017.

9. Zhou, Z.; Redaelli, A.; Johnell, O.; Willke, R.J.; Massimini, G. A retrospective analysis of health care costs for bone fractures in women with early-stage breast carcinoma. Cancer 2004, 100, 507-517. [CrossRef]

10. Pajarinen, J.; Lin, T.; Gibon, E.; Kohno, Y.; Maruyama, M.; Nathan, K.; Lu, L.; Yao, Z.; Goodman, S.B. Mesenchymal stem cell-macrophage crosstalk and bone healing. Biomaterials 2018. [CrossRef]

11. Kojouharov, H.V.; Trejo, I.; Chen-Charpentier, B.M. Modeling the effects of inflammation in bone fracture healing. AIP Conf. Proc. 2017, 1895, 020005.

12. Schlundt, C.; El Khassawna, T.; Serra, A.; Dienelt, A.; Wendler, S.; Schell, H.; van Rooijen, N.; Radbruch, A.; Lucius, R.; Hartmann, S.; et al. Macrophages in bone fracture healing: Their essential role in endochondral ossification. Bone 2018, 106, 78-89. [CrossRef] [PubMed]

13. Zhang, R.; Liang, Y.; Wei, S. M2 macrophages are closely associated with accelerated clavicle fracture healing in patients with traumatic brain injury: A retrospective cohort study. J. Orthop. Surg. Res. 2018, 13, 213. [CrossRef]

14. Marsell, R.; Einhorn, T.A. The biology of fracture healing. Injury 2011, 42, 551-555. [CrossRef] [PubMed]

15. Chung, E.; Son, Y. Crosstalk between mesenchymal stem cells and macrophages in tissue repair. Tissue Eng. Regen. Med. 2014, 11, 431-438. [CrossRef]

16. Schlundt, C.; Schell, H.; Goodman, S.B.; Vunjak-Novakovic, G.; Duda, G.N.; Schmidt-Bleek, K. Immune modulation as a therapeutic strategy in bone regeneration. J. Exp. Orthop. 2015, 2, 1. [CrossRef] [PubMed]

17. Chan, J.K.; Glass, G.E.; Ersek, A.; Freidin, A.; Williams, G.A.; Gowers, K.; Santo, A.I.E.; Jeffery, R.; Otto, W.R.; Poulsom, R.; et al. Low-dose TNF augments fracture healing in normal and osteoporotic bone by up-regulating the innate immune response. EMBO Mol. Med. 2015, 7, 547-561. [CrossRef] [PubMed]

18. Mantovani, A.; Sozzani, S.; Locati, M.; Allavena, P.; Sica, A. Macrophage polarization: Tumor-associated macrophages as a paradigm for polarized M2 mononuclear phagocytes. Trends Immunol. 2002, 23, 549-555. [CrossRef]

19. Border, W.A.; Noble, N.A. Transforming growth factor $\beta$ in tissue fibrosis. N. Engl. J. Med. 1994, 331, 1286-1292.

20. Cavaillon, J.M. Pro-versus anti-inflammatory cytokines: Myth or reality. Cell. Mol. Biol. 2001, 47, 695-702.

21. Osta, B.; Benedetti, G.; Miossec, P. Classical and paradoxical effects of TNF- $\alpha$ on bone homeostasis. Front. Immunol. 2014, 5, 48. [CrossRef] 
22. Arango Duque, G.; Descoteaux, A. Macrophage cytokines: Involvement in immunity and infectious diseases. Front. Immunol. 2014, 5, 491. [CrossRef] [PubMed]

23. Mountziaris, P.M.; Mikos, A.G. Modulation of the inflammatory response for enhanced bone tissue regeneration. Tissue Eng. Part B Rev. 2008, 14, 179-186. [CrossRef] [PubMed]

24. Gibon, E.; Lu, L.Y.; Nathan, K.; Goodman, S.B. Inflammation, ageing, and bone regeneration. J. Orthop. Transl. 2017, 10, 28-35. [CrossRef] [PubMed]

25. Gibon, E.; Loi, F.; Córdova, L.A.; Pajarinen, J.; Lin, T.; Lu, L.; Nabeshima, A.; Yao, Z.; Goodman, S.B. Aging affects bone marrow macrophage polarization: Relevance to bone healing. Regen. Eng. Transl. Med. 2016, 2, 98-104. [CrossRef] [PubMed]

26. Serhan, C.N.; Savill, J. Resolution of inflammation: The beginning programs the end. Nat. Immunol. 2005, 6, 1191-1197. [CrossRef] [PubMed]

27. Newman, S.L.; Henson, J.E.; Henson, P.M. Phagocytosis of senescent neutrophils by human monocyte-derived macrophages and rabbit inflammatory macrophages. J. Exp. Med. 1982, 156, 430-442. [CrossRef]

28. Italiani, P.; Boraschi, D. From monocytes to M1/M2 macrophages: Phenotypical vs. functional differentiation. Front. Immunol. 2014, 5, 514. [CrossRef]

29. Wang, Y.; Yang, T.; Ma, Y.; Halade, G.V.; Zhang, J.; Lindsey, M.L.; Jin, Y.F. Mathematical modeling and stability analysis of macrophage activation in left ventricular remodeling post-myocardial infarction. BMC Genom. 2012, 13, S21. [CrossRef]

30. Bailon-Plaza, A.; Van Der Meulen, M.C. A mathematical framework to study the effects of growth factor influences on fracture healing. J. Theor. Biol. 2001, 212, 191-209. [CrossRef]

31. Echeverri, L.; Herrero, M.; Lopez, J.; Oleaga, G. Early stages of bone fracture healing: Formation of a fibrin-collagen scaffold in the fracture hematoma. Bull. Math. Biol. 2015, 77, 156-183. [CrossRef]

32. Doblaré, M.; Garcia, J.; Gómez, M. Modelling bone tissue fracture and healing: A review. Eng. Fract. Mech. 2004, 71, 1809-1840. [CrossRef]

33. Ghiasi, M.S.; Chen, J.; Vaziri, A.; Rodriguez, E.K.; Nazarian, A. Bone fracture healing in mechanobiological modeling: A review of principles and methods. Bone Rep. 2017, 6, 87-100. [CrossRef] [PubMed]

34. Einhorn, T.A. The science of fracture healing. J. Orthop. Trauma 2005, 19, S4-S6. [CrossRef] [PubMed]

35. Einhorn, T.A.; Gerstenfeld, L.C. Fracture healing: Mechanisms and interventions. Nat. Rev. Rheumatol. 2015, 11, 45. [CrossRef] [PubMed]

36. Gómez-Barrena, E.; Rosset, P.; Lozano, D.; Stanovici, J.; Ermthaller, C.; Gerbhard, F. Bone fracture healing: Cell therapy in delayed unions and nonunions. Bone 2015, 70, 93-101. [CrossRef] [PubMed]

37. Carlier, A.; Geris, L.; van Gastel, N.; Carmeliet, G.; Van Oosterwyck, H. Oxygen as a critical determinant of bone fracture healing-A multiscale model. J. Theor. Biol. 2015, 365, 247-264. [CrossRef] [PubMed]

38. Nagaraja, S.; Wallqvist, A.; Reifman, J.; Mitrophanov, A.Y. Computational approach to characterize causative factors and molecular indicators of chronic wound inflammation. J. Immunol. 2014, 192, 1824-1834. [CrossRef] [PubMed]

39. Yu, T. Design and Validation of A Mathematical Model to Describe Macrophage Dynamics in Wound Healing. Ph.D. Thesis, Drexel University, Philadelphia, PA, USA, 2014.

40. Otto, S.P.; Day, T. A Biologist's Guide to Mathematical Modeling in Ecology and Evolution; Princeton University Press: Princeton, NJ, USA, 2011.

41. Marée, A.F.; Komba, M.; Dyck, C.; Labkecki, M.; Finegood, D.T.; Edelstein-Keshet, L. Quantifying macrophage defects in type 1 diabetes. J. Theor. Biol. 2005, 233, 533-551. [CrossRef]

42. Sherratt, J.A.; Murray, J. Models of epidermal wound healing. Proc. R. Soc. Lond. B 1990, 241, $29-36$.

43. Isaksson, H. Recent advances in mechanobiological modeling of bone regeneration. Mech. Res. Commun. 2012, 42, 22-31. [CrossRef]

44. Schell, H.; Duda, G.; Peters, A.; Tsitsilonis, S.; Johnson, K.; Schmidt-Bleek, K. The haematoma and its role in bone healing. J. Exp. Orthop. 2017, 4, 5. [CrossRef] [PubMed]

45. Van Furth, R.; Diesselhoff-den Dulk, M.M.; Mattie, H. Quantitative study on the production and kinetics of mononuclear phagocytes during an acute inflammatory reaction. J. Exp. Med. 1973, 138, 1314-1330. [CrossRef] [PubMed] 
46. Bastidas-Coral, A.P.; Bakker, A.D.; Zandieh-Doulabi, B.; Kleverlaan, C.J.; Bravenboer, N.; Forouzanfar, T.; Klein-Nulend, J. Cytokines TNF- $\alpha$, IL-6, IL-17F, and IL-4 differentially affect osteogenic differentiation of human adipose stem cells. Stem Cells Int. 2016, 2016, 1318256. [CrossRef] [PubMed]

47. Gilbert, L.; He, X.; Farmer, P.; Boden, S.; Kozlowski, M.; Rubin, J.; Nanes, M.S. Inhibition of Osteoblast Differentiation by Tumor Necrosis Factor- $\alpha$ 1. Endocrinology 2000, 141, 3956-3964. [CrossRef] [PubMed]

48. Lacey, D.; Simmons, P.; Graves, S.; Hamilton, J. Proinflammatory cytokines inhibit osteogenic differentiation from stem cells: Implications for bone repair during inflammation. Osteoarthritis Cartilage 2009, 17, 735-742. [CrossRef] [PubMed]

49. Lu, Y.; Lekszycki, T. Modeling of an initial stage of bone fracture healing. Contin. Mech. Thermodyn. 2015, 27, 851. [CrossRef]

50. Hoegel, F.; Abdulazim, A.; Augat, P.; Buehren, V. Quantification of reaming debris at the fracture gap of diaphyseal A2 and A3 Fractures After reamed intramedullary nailing of the sheep tibia. Eur. J. Trauma Emerg. Surg. 2008, 34, 587-591. [CrossRef]

51. Gaston, M.; Simpson, A. Inhibition of fracture healing. Bone Joint J. 2007, 89, 1553-1560. [CrossRef]

52. Roberts, T.T.; Rosenbaum, A.J. Bone grafts, bone substitutes and orthobiologics: The bridge between basic science and clinical advancements in fracture healing. Organogenesis 2012, 8, 114-124. [CrossRef]

53. Wu, M.; Chen, G.; Li, Y.P. TGF- $\beta$ and BMP signaling in osteoblast, skeletal development, and bone formation, homeostasis and disease. Bone Res. 2016, 4, 16009. [CrossRef]

54. Recknagel, S.; Bindl, R.; Brochhausen, C.; Göckelmann, M.; Wehner, T.; Schoengraf, P.; Huber-Lang, M.; Claes, L.; Ignatius, A. Systemic inflammation induced by a thoracic trauma alters the cellular composition of the early fracture callus. J. Trauma Acute Care Surg. 2013, 74, 531-537. [CrossRef] [PubMed]

55. Stuart, A.; Humphries, A.R. Dynamical Systems and Numerical Analysis; Cambridge University Press: Cambridge, UK, 1998.

56. Strang, G. Introduction to Linear Algebra; Wellesley-Cambridge Press: Wellesley, MA, USA, 1993.

(C) 2019 by the authors. Licensee MDPI, Basel, Switzerland. This article is an open access article distributed under the terms and conditions of the Creative Commons Attribution (CC BY) license (http:/ / creativecommons.org/licenses/by/4.0/). 